J. Nonlinear Var. Anal. 5 (2021), No. 2, pp. 227-250

Available online at http://jnva.biemdas.com

https://doi.org/10.23952/jnva.5.2021.2.04

\title{
SOME RESULTS ASSOCIATED TO FIRST-ORDER SET-VALUED EVOLUTION PROBLEMS WITH SUBDIFFERENTIALS
}

\author{
SOUMIA SAÏDI \\ LMPA Laboratory, Department of Mathematics, Mohamed Seddik Ben Yahia University, Jijel, Algeria
}

\begin{abstract}
In Hilbert spaces, we establish some results related to a class of first-order differential inclusions governed by time-dependent subdifferential operators. Based on the existence result, we study a system governed by a couple of an evolution inclusion involving the time-dependent subdifferential operator and a differential equation with the Caputo fractional derivative.
\end{abstract}

Keywords. Differential inclusion; Subdifferential; Maximal monotone operator; Caputo fractional derivative; Fixed point.

\section{INTRODUCTION}

Differential inclusions involving time-dependent subdifferential (denoted $\partial \varphi$ ) operators have been developed recently. Several results are known under various conditions expressed in terms of $\varphi$, the Fenchel conjugate function $\varphi^{*}(t, \cdot)$, the Yosida approximation of $\partial \varphi(t, \cdot)$, or the Moreau envelope $\varphi_{\lambda}(t, \cdot)$. In particular, sweeping processes are the differential inclusions governed by the subdifferential of the indicator function of a moving convex (or $r$-prox regular) set depending on time $C(t)$ (or on both time and state $C(t, x)$ ) (see, e.g., [1]). The evolution problems governed by subdifferential operators and sweeping processes were motivated by the elastoplaticity and the friction dynamics. They have various applications in nonsmooth mechanics (see [2]), economics $([3,4])$, electrical circuits (see [5]), crowd motion modeling ([6]), and so on. The existence and uniqueness of solutions to such systems and their classical variants (subjected to perturbation forces, prox-regular state dependent set $C(t, x), \ldots$ ) have been thoroughly studied by many authors recently. For some contributions on the first-order differential inclusions governed by subdifferential operators and sweeping processes, we refer to [7]-[22] and the references therein. For the more general time-dependent maximal monotone operators case, the reader is referred to $[23,24,25,26]$.

Peralba is one of the pioneers in the study of the differential inclusions governed by timedependent subdifferential operators. In [18], it was used an assumption expressed in term of the conjugate function $\varphi^{*}(t, \cdot)$ (see assumption $\left(\mathrm{H}_{2}\right)$ below). This assumption has been extensively adopted by several authors; see e.g. $[8,10,12,19,20]$ and the references therein.

${ }^{*}$ Corresponding author.

E-mail address: soumiasaidi44@gmail.com.

Received February 6, 2020; Accepted January 29, 2021.

(C)2021 Journal of Nonlinear and Variational Analysis 
We are concerned here, with a new variant by considering a natural generalization of the differential inclusion involving time-dependent subdifferential. This variant is of the form

$$
\left\{\begin{array}{l}
-\dot{x}(t) \in \partial \varphi(t, x(t))+F(t, x(t))+f(t, x(t)) \quad \text { a.e. } t \in I, \\
x\left(T_{0}\right)=x_{0} \in \operatorname{dom} \varphi\left(T_{0}, \cdot\right),
\end{array}\right.
$$

where $f$ (resp. $F$ ) is a single-valued (resp. set-valued) map. The current work covers many results on the perturbed convex sweeping process or the Moreau process (see, e.g., [2, 27, 28, 29]). The interest to such a variant is also motivated by the results on the perturbed sweeping process related to our evolution problem that has been recently studied, i.e., for $\varphi(t, \cdot)$ taken as the indicator function of a non-empty $r$-prox regular moving subset $C(t)$ of $H$, see [30] in the absolutely continuous framework. Several related results have been developed in [31] and [32] for the case that the sets $C(t)$ are uniformly prox-regular and move with bounded variation. Dealing with time-dependent maximal monotone operator $A(t)$ instead of $\partial \varphi(t, \cdot)$ in the variant above, we refer to [23].

We will proceed, to state the main existence theorem, by a discretisation method. We solve at each subinterval a problem involving single-valued time-dependent perturbation by making use of the results presented in $[18,19]$. The limit of a sequence of functions is proved to be a solution to our problem.

This paper consists of six sections. In Section 2, we give notation and the necessary background materials. In Section 3, we summarize some results from [18] and [19]. In Section 4, we propose the existence result to our problem. The approach to our main theorem of this section is based on the measurability result of [33] concerning the element of minimal norm. In Section 5, we are interested in a coupled problem by a fractional differential equation with the evolution inclusion studied in the previous section. In Section 6, the last section, a concluding remark is provided.

\section{PRELIMinARIES}

Throughout the paper, we use $I:=\left[T_{0}, T\right]$ to denote an interval of $\mathbb{R}$ and use $H$ to stand for a real Hilbert space whose inner product is denoted by $\langle\cdot, \cdot\rangle$ and the associated norm by $\|\cdot\|$. We denote by $\bar{B}_{H}$ the closed unit ball. Let $S_{1}, S_{2}$ be two subsets of $H$. Define the Hausdorff distance between $S_{1}$ and $S_{2}$ by

$$
d_{H}\left(S_{1}, S_{2}\right)=\max \left(\sup _{x \in S_{2}} d\left(x, S_{1}\right), \sup _{x \in S_{1}} d\left(x, S_{2}\right)\right)
$$

where $d(x, S)=\inf _{y \in S}\|x-y\|$, for any subset $S$ of $H$. For any interval $I$ of $\mathbb{R}$, we denote by $\mathscr{L}(I)$ (resp. $\mathscr{B}(H)$ ) the $\sigma$-algebra of measurable sets of $I$ (resp. Borel $\sigma$-algebra of measurable sets of $H$ ). On the space $\mathscr{C}_{H}(I)$ of continuous maps $x: I \rightarrow H$, we consider the norm of the uniform convergence on $I,\|x\|_{\infty}=\sup _{t \in I}\|x(t)\|$. By $L_{H}^{p}(I)$ for $p \in[1,+\infty[($ resp. $p=+\infty)$, we denote the space of measurable maps $x: I \rightarrow H$ such that $\int_{I}\|x(t)\|^{p} d t<+\infty$ (resp. which are essentially bounded) endowed with the usual norm $\|x\|_{L_{H}^{p}(I)}=\left(\int_{I}\|x(t)\|^{p} d t\right)^{\frac{1}{p}}, 1 \leq p<+\infty$ (resp. endowed with the usual essential supremum norm $\left.\|\cdot\|_{L_{H}^{\infty}(I)}\right)$. By $W^{1,2}(I, H)$, we denote the space of absolutely continuous functions from $I$ to $H$ with derivatives in $L_{H}^{2}(I)$. 
Let $\varphi$ be a lower semi-continuous convex function from $H$ to $\mathbb{R} \cup\{+\infty\}$, which is proper in the sense that its effective domain $(\operatorname{dom} \varphi)$ defined by

$$
\operatorname{dom} \varphi=\{x \in H: \varphi(x)<+\infty\}
$$

is non-empty. As usual, its Fenchel conjugate is defined by

$$
\varphi^{*}(v)=\sup _{x \in H}[\langle v, x\rangle-\varphi(x)] .
$$

The subdifferential $\partial \varphi(x)$ of $\varphi$ at $x \in \operatorname{dom} \varphi$ is

$$
\partial \varphi(x)=\{v \in H: \varphi(y) \geq\langle v, y-x\rangle+\varphi(x) \forall y \in \operatorname{dom} \varphi\}
$$

and its effective domain is $\operatorname{Dom} \partial \varphi=\{x \in H: \partial \varphi(x) \neq \emptyset\}$. It is well known (see, e.g., [9]) that if $\varphi$ is a proper lower semi-continuous convex function, then its subdifferential operator $\partial \varphi$ is a maximal monotone operator.

Let $S$ be a non-empty closed convex subset of $H$. Define the projection of a point $x \in H$ onto $S$ by $P_{S}(x)=\{y \in S:\|x-y\|=d(x, S)\}$. Denote by $\psi_{S}$ the indicator function of $S$, that is, $\psi_{S}(x)=0$ if $x \in S$ and $+\infty$ otherwise; and by $\delta^{*}(\cdot, S)$ the support function of $S$ defined by

$$
\delta^{*}(x, S)=\sup _{y \in S}\langle y, x\rangle \text { for all } x \in H .
$$

From the definition of $\delta^{*}(\cdot, S)$, we deduce that $\delta^{*}(\cdot, S)$ coincides with the Fenchel conjugate of $\psi_{S}$, that is, $\delta^{*}(\cdot, S)=\left(\psi_{S}\right)^{*}$. Denote by $N_{S}(x)$ the normal cone to $S$ at $x \in H$, which is the subdifferential of the indicator function of $S$ at $x$, that is,

$$
N_{S}(x)=\partial \psi_{S}(x)=\{y \in H:\langle y, z-x\rangle \leq 0 \forall z \in S\},
$$

where $N_{S}(x)$ is a maximal monotone operator. Let $F: E \rightrightarrows H$ be a set-valued map from a Hausdorff topological space $E$ into $H$. The set-valued map $F$ is said to be scalarly upper semicontinuous if, for any $y \in H$, the real-valued function $x \mapsto \delta^{*}(F(x), y)$ is upper semicontinuous. Let $F: T \rightrightarrows Y$ be a set-valued map from a measurable space $(T, \mathscr{T})$ into a metric space $Y$. The set-valued map $F$ is measurable if, for any open set $U$ of $Y$, we have $F^{-1}(U) \in \mathscr{T}$, with $F^{-1}(U)=\{t \in T: F(t) \cap U \neq \emptyset\}$. For more details on the properties of maximal monotone operators in Hilbert spaces, we refer to [9]. We also refer to [33] for details concerning convex analysis and measurable set-valued-maps.

\section{Auxiliary Results}

To start this section, we give an overview concerning the assumptions on the function $\varphi(\cdot, \cdot)$ occurring in the subdifferential operator used in the paper.

3.1. Assumptions and connections to the sweeping process. The first-order time-dependent sweeping process has the form

$$
\left\{\begin{aligned}
-\dot{x}(t) & \in N_{C(t)}(x(t)) \quad \text { a.e. } t \in[0, T], \\
x(0) & =x_{0} \in C(0),
\end{aligned}\right.
$$

where $N_{C(t)}=\partial \psi_{C(t)}$ is defined by (2.1). This problem has been introduced and extensively studied in the period of 1970s by Moreau in a series of seminal papers (see, e.g., [1, 28, 34]). 
In [1], Moreau involved closed convex sets $C(t)$ that vary in an absolutely continuous way, that is, there exists an absolutely continuous function $a: I \rightarrow \mathbb{R}$ such that

$$
\left|\delta^{*}(x, C(t))-\delta^{*}(x, C(s))\right| \leq\|x\||| a(t)-a(s) \mid,
$$

for any $x \in H$ and any $t, s \in[0, T]$. To handle the first-order differential inclusions governed by subdifferential operators of the form

$$
\left\{\begin{array}{l}
-\dot{x}(t) \in \partial \varphi(t, x(t)) \quad \text { a.e. } t \in[0, T] \\
x\left(T_{0}\right)=x_{0} \in \operatorname{dom} \varphi(0, \cdot)
\end{array}\right.
$$

Peralba [18] considered the following assumptions:

$\left(H_{1}\right)$ for each $t \in I$, the function $x \mapsto \varphi(t, x)$ is proper, lower semi-continuous, and convex;

$\left(H_{2}\right)$ there exist a $\rho$-Lipschitz function $k: H \longrightarrow \mathbb{R}_{+}$and an absolutely continuous function $a: I \rightarrow \mathbb{R}$, with a derivative $\dot{a} \in L_{\mathbb{R}}^{2}(I)$, such that

$$
\varphi^{*}(t, x) \leq \varphi^{*}(s, x)+k(x)|a(t)-a(s)| \text { for every }(t, s, x) \in I \times I \times H .
$$

We will establish our main results under $\left(H_{1}\right)-\left(H_{2}\right)$ with the inequality $\dot{a}(\cdot) \geq 0$ in condition $\left(H_{2}\right)$. Note that if condition $\left(H_{2}\right)$ is satisfied with an absolutely continuous function $a(\cdot)$ for which the latter inequality is not fulfilled by the derivative, it is easily seen that it is still satisfied with the absolutely continuous function $t \mapsto \int_{T_{0}}^{t}|\dot{a}(\tau)| d \tau$ whose derivative is non-negative.

3.2. The problems governed by the subdifferential operators with time-dependent single valued-perturbation. First, let us recall an existence and uniqueness result of Peralba [18] (see also [19]).

Theorem 3.1. Let $\varphi: I \times H \rightarrow[0,+\infty]$ be a map satisfying $\left(H_{1}\right)-\left(H_{2}\right)$. Let $x_{0} \in \operatorname{dom} \varphi\left(T_{0}, \cdot\right)$ be fixed. Then, the differential inclusion

$$
\left\{\begin{array}{c}
-\dot{x}(t) \in \partial \varphi(t, x(t)) \quad \text { a.e. } t \in I, \\
x\left(T_{0}\right)=x_{0} \in \operatorname{dom} \varphi\left(T_{0}, \cdot\right),
\end{array}\right.
$$

has a unique absolutely continuous solution $x(\cdot)$ on I. Moreover, $x(t) \in \operatorname{dom} \varphi(t, \cdot)$ for all $t \in I$, and the following estimate holds

$$
\|\dot{x}\|_{L_{H}^{2}(I)} \leq \frac{\rho}{2}\|\dot{a}\|_{L_{\mathbb{R}}^{2}(I)}+\left[\sqrt{T-T_{0}} k(0)\|\dot{a}\|_{L_{\mathbb{R}}^{2}(I)}+\frac{\rho^{2}}{4}\|\dot{a}\|_{L_{\mathbb{R}}^{2}(I)}^{2}+\varphi\left(T_{0}, x_{0}\right)-\varphi(T, x(T))\right]^{\frac{1}{2}} .
$$

Remark 3.1. Let $C: I \rightrightarrows H$ be a set-valued map such that $\left(H_{1}^{\prime}\right)$ for each $t \in I, C(t)$ is a non-empty convex closed subset of $H$;

$\left(H_{2}^{\prime}\right)$ there exists an absolutely continuous function $a: I \rightarrow \mathbb{R}$, with a derivative $\dot{a} \in L_{\mathbb{R}}^{2}(I)$, such that

$$
d_{H}(C(t), C(s)) \leq|a(t)-a(s)| \forall t, s \in I .
$$

From Hormander formula (see [33]), one gets

$$
\left|\delta^{*}(x, C(t))-\delta^{*}(x, C(s))\right| \leq\|x\| d_{H}(C(t), C(s)) \leq\|x\||a(t)-a(s)|,
$$

for any $x \in H$ and any $t, s \in I$. Set $\varphi(t, x)=\psi_{C(t)}(x)$ for any $(t, x) \in I \times H$. It is easy to check that hypothesis $\left(H_{1}\right)-\left(H_{2}\right)$ are satisfied. In view of Theorem 3.1, one sees that there exists a unique absolutely continuous solution to the sweeping process

$$
\left\{\begin{array}{c}
-\dot{x}(t) \in N_{C(t)}(x(t)) \quad \text { a.e. } t \in I, \\
x\left(T_{0}\right)=x_{0} \in C\left(T_{0}\right) .
\end{array}\right.
$$


Now, we denote by $A(t):=\partial \varphi(t, \cdot)$ the maximal monotone operator in $H$ associated with $\partial \varphi(t, \cdot), t \in I\left(\varphi\right.$ satisfies conditions $\left.\left(H_{1}\right)-\left(H_{2}\right)\right)$. Let us consider the operator $\mathscr{A}: L_{H}^{2}(I) \rightrightarrows L_{H}^{2}(I)$ defined by

$$
\mathscr{A} x=\left\{y \in L_{H}^{2}(I): y(t) \in A(t) x(t) \text { a.e. }\right\} .
$$

Then, $\mathscr{A}$ is well defined since the evolution inclusion

$$
-\dot{x}(t) \in A(t) x(t)=\partial \varphi(t, x(t)) \text { a.e. } t \in I, x_{0}=x\left(T_{0}\right) \in \operatorname{dom} \varphi\left(T_{0}, \cdot\right),
$$

admits a unique absolutely continuous solution due to Theorem 3.1.

The operator $\mathscr{A}$ enjoys the following property; see [18] (see also [12]).

Proposition 3.1. Assume that, for any $t \in I, A(t)=\partial \varphi(t, \cdot)$, where $\varphi$ satisfies conditions $\left(H_{1}\right)$ $\left(\mathrm{H}_{2}\right)$. Then,

$(\mathscr{J})$ the operator $\mathscr{A}$ is maximal monotone;

$(\mathscr{J} \mathscr{J})$ if $\left(x_{n}\right)_{n}$ and $\left(y_{n}\right)_{n}$ are two sequences in $L_{H}^{2}(I)$ satisfying

(i) $y_{n}(t) \in A(t) x_{n}(t), \forall n \in \mathbb{N}$ and for a.e. $t \in I$;

(ii) $\left(x_{n}\right)_{n}$ strongly converges to $x$ in $L_{H}^{2}(I)$;

(iii) $\left(y_{n}\right)_{n}$ weakly converges to $y$ in $L_{H}^{2}(I)$;

then, $y(t) \in A(t) x(t)$ a.e. $t \in I$.

Proof. Since $(\mathscr{J})$ is clear, we omit the proof here. Let us prove assertion $(\mathscr{J} \mathscr{J})$. By $(i)$, one has $y_{n} \in \mathscr{A} x_{n}$, where $\mathscr{A}$ is the maximal monotone operator associated with $A(t)$ (see $(\mathscr{J})$ ). Since the graph of $\mathscr{A}$ is sequentially strongly-weakly closed, by $y_{n} \in \mathscr{A} x_{n}$ and by (ii) and (iii), one deduces that $y \in \mathscr{A} x$. Hence, from the definition of $\mathscr{A}$, one gets $y(t) \in A(t) x(t)$ a.e. $t \in I$.

We will need the following useful application of Theorem 3.1 concerning an evolution problem with single-valued perturbation depending only on time; see [19, Proposition 3.4].

Proposition 3.2. Under the assumptions of Theorem 3.1, if $h \in L_{H}^{2}(I)$ and $x_{0} \in \operatorname{dom} \varphi\left(T_{0}, \cdot\right)$, then the evolution problem

$$
\left\{\begin{array}{c}
-\dot{x}(t) \in \partial \varphi(t, x(t))+h(t) \quad \text { a.e. } t \in I \\
x\left(T_{0}\right)=x_{0} \in \operatorname{dom} \varphi\left(T_{0}, \cdot\right)
\end{array}\right.
$$

admits a unique absolutely continuous solution $x(\cdot)$ satisfying

$$
\|\dot{x}\|_{L_{H}^{2}(I)}^{2} \leq \sigma\|h\|_{L_{\mathbb{R}}^{2}(I)}^{2}+\eta
$$

where $\eta$ and $\sigma$ are non-negative real constants defined by

$$
\begin{aligned}
\eta & =\left[3(\rho+1)^{2}+k^{2}(0)\right]\|\dot{a}\|_{L_{\mathbb{R}}^{2}(I)}^{2}+2\left[T-T_{0}+\varphi\left(T_{0}, x_{0}\right)-\varphi(T, x(T))\right], \\
\sigma & =3(\rho+1)^{2}+k^{2}(0)+4 .
\end{aligned}
$$

Proof. Set $\Phi(t)=\int_{T_{0}}^{t} h(s) d s$ for every $t \in I$. Then, the differential inclusion

$$
\left\{\begin{array}{c}
-\dot{x}(t) \in \partial \varphi(t, x(t))+h(t) \quad \text { a.e. } t \in I, \\
x\left(T_{0}\right)=x_{0} \in \operatorname{dom} \varphi\left(T_{0}, \cdot\right)
\end{array}\right.
$$

is equivalent to the following one

$$
\left\{\begin{array}{c}
-\dot{x}_{1}(t) \in \partial \varphi_{1}\left(t, x_{1}(t)\right) \quad \text { a.e. } t \in I \\
x_{1}\left(T_{0}\right)=x_{0} \in \operatorname{dom} \varphi_{1}\left(T_{0}, \cdot\right)
\end{array}\right.
$$


where

$$
\begin{aligned}
x_{1}(t) & =x(t)+\Phi(t), \forall t \in I, \\
\varphi_{1}(t, z) & =\varphi(t, z-\Phi(t)), \forall(t, z) \in I \times H .
\end{aligned}
$$

Therefore, it is sufficient to show that $\varphi_{1}$ satisfies the conditions of Theorem 3.1. It is obvious that, for every $t \in I$, the function $\varphi_{1}(t, \cdot)$ is non-negative, proper, convex, and lower semi-continuous on $H$. So, it remains to examine the corresponding properties of the conjugate function $\varphi_{1}^{*}(t, \cdot)$ of $\varphi_{1}(t, \cdot)$. Note that

$$
\varphi_{1}^{*}(t, z)=\varphi^{*}(t, z)+\langle z, \Phi(t)\rangle,
$$

for all $(t, z) \in I \times H$. Consequently, one has

$$
\varphi_{1}^{*}(t, z) \leq \varphi_{1}^{*}(s, z)+k(z)|a(t)-a(s)|+\|z\|\|\Phi(t)-\Phi(s)\|,
$$

for all $(t, s, z) \in I \times I \times H$. Now, setting

$$
\begin{aligned}
& k_{1}(z)=k(z)+\|z\|, \forall z \in H \\
& a_{1}(t)=\int_{T_{0}}^{t}[\dot{a}(\tau)+\|h(\tau)\|] d \tau, \forall t \in I,
\end{aligned}
$$

we have that $k_{1}$ is $(\rho+1)$-Lipschitz, and $a_{1}$ is absolutely continuous with $\dot{a}_{1} \in L_{\mathbb{R}}^{2}(I)$. Hence, $\varphi_{1}^{*}$ satisfies

$$
\varphi_{1}^{*}(t, z) \leq \varphi_{1}^{*}(s, z)+k_{1}(z)\left|a_{1}(t)-a_{1}(s)\right|,
$$

for all $(t, s, z) \in I \times I \times H$. Applying Theorem 3.1, we see that the perturbed problem above has a unique solution $x_{1}$ such that the following estimate

$$
\begin{aligned}
\left\|\dot{x}_{1}\right\|_{L_{H}^{2}(I)} \leq & \frac{\rho+1}{2}\left\|\dot{a}_{1}\right\|_{L_{\mathbb{R}}^{2}(I)}+\left[\sqrt{T-T_{0}} k(0)\left\|\dot{a}_{1}\right\|_{L_{\mathbb{R}}^{2}(I)}\right. \\
& \left.+\frac{(\rho+1)^{2}}{4}\left\|\dot{a}_{1}\right\|_{L_{\mathbb{R}}^{2}(I)}^{2}+\varphi_{1}\left(T_{0}, x_{0}\right)-\varphi_{1}\left(T, x_{1}(T)\right)\right]^{\frac{1}{2}},
\end{aligned}
$$

holds true (due to $k_{1}(0)=k(0)$ ). It follows that

$$
\begin{aligned}
\|\dot{x}\|_{L_{H}^{2}(I)} \leq & \frac{1}{2}(\rho+1)\|\dot{a}+|h|\|_{L_{\mathbb{R}}^{2}(I)}+\|h\|_{L_{H}^{2}(I)} \\
& +\left[\sqrt{T-T_{0}} k(0)\|\dot{a}+|h|\|_{L_{\mathbb{R}}^{2}(I)}+\frac{(\rho+1)^{2}}{4}\|\dot{a}+|h|\|_{L_{\mathbb{R}}^{2}(I)}^{2}+\varphi\left(T_{0}, x_{0}\right)-\varphi(T, x(T))\right]^{\frac{1}{2}},
\end{aligned}
$$

where $|h|$ is the function of $I$ into $\mathbb{R}$ defined by $|h|(t):=\|h(t)\|$ for all $t \in I$. Now, by using the inequality $2 e f \leq e^{2}+f^{2}$, for all $e, f \in \mathbb{R}$, we have

$$
\begin{aligned}
\sqrt{T-T_{0}} k(0)\|\dot{a}+|h|\|_{L_{\mathbb{R}}^{2}(I)} & =2 \sqrt{T-T_{0}}\left(\frac{k(0)}{2}\|\dot{a}+|h|\|_{L_{\mathbb{R}}^{2}(I)}\right) \\
& \leq\left(T-T_{0}\right)+\frac{k^{2}(0)}{4}\|\dot{a}+|h|\|_{L_{\mathbb{R}}^{2}(I)}^{2},
\end{aligned}
$$

which implies that

$$
\begin{gathered}
\|\dot{x}\|_{L_{H}^{2}(I)} \leq \frac{(\rho+1)}{2}\|\dot{a}+|h|\|_{L_{\mathbb{R}}^{2}(I)}+\|h\|_{L_{H}^{2}(I)} \\
+\left[\left(T-T_{0}\right)+\frac{\left(k^{2}(0)+(\rho+1)^{2}\right)}{4}\|\dot{a}+\mid h\|_{L_{\mathbb{R}}^{2}(I)}^{2}+\varphi\left(T_{0}, x\left(T_{0}\right)\right)-\varphi(T, x(T))\right]^{\frac{1}{2}}
\end{gathered}
$$


By using the inequality $(e+f)^{2} \leq 2\left(e^{2}+f^{2}\right)$, for all $e, f \in \mathbb{R}$, we conclude that

$$
\begin{aligned}
\|\dot{x}\|_{L_{H}^{2}(I)}^{2} \leq & 2\left[\frac{(\rho+1)}{2}\|\dot{a}+|h|\|_{L_{\mathbb{R}}^{2}(I)}+\|h\|_{L_{H}^{2}(I)}\right]^{2} \\
& +2\left[\left(T-T_{0}\right)+\frac{\left(k^{2}(0)+(\rho+1)^{2}\right)}{4}\|\dot{a}+\mid h\|_{L_{\mathbb{R}}^{2}(I)}^{2}+\varphi\left(T_{0}, x\left(T_{0}\right)\right)-\varphi(T, x(T))\right] .
\end{aligned}
$$

Simplifying the right member of the last inequality yields (3.2) immediately.

\section{MAin Results}

The following assumptions are used in the sequel. Let $f: I \times H \rightarrow H$ be a Carathéodory map such that there exists a non-negative function $\alpha(\cdot) \in L_{\mathbb{R}}^{2}(I)$ such that, for all $t \in I$ and for all $x \in H$,

$$
\|f(t, x)\| \leq \alpha(t)(1+\|x\|) .
$$

Let $F: I \times H \rightrightarrows H$ be a set-valued map with non-empty convex weakly compact values satisfying

(j) $F$ is scalarly $\mathscr{L}(I) \otimes \mathscr{B}(H)$-measurable, that is, for any $y \in H$, the function $\delta^{*}(y, F(\cdot, \cdot))$ is $\mathscr{L}(I) \otimes \mathscr{B}(H)$-measurable;

$(j j)$ for any $t \in I, F(t, \cdot)$ is scalarly upper semi-continuous, that is, for any $y \in H$, the function $\delta^{*}(y, F(t, \cdot))$ is upper semi-continuous on $H$;

$(j j j)$ for some $\beta \in L_{\mathbb{R}_{+}}^{2}(I), F$ satisfies the following condition

$$
d(0, F(t, x)) \leq \beta(t)(1+\|x\|) \bar{B}_{H} \text { for all }(t, x) \in I \times H .
$$

It is worth to emphasize that the method used to prove our first theorem was introduced in [19].

Theorem 4.1. Let $H$ be a real separable Hilbert space. Let $\varphi: I \times H \rightarrow[0,+\infty]$ be a map satisfying $\left(H_{1}\right)-\left(H_{2}\right)$, and the fact that $\operatorname{dom} \varphi(t, \cdot)$ is ball-compact for every $t \in I$. Let $f$ : $I \times H \rightarrow H$ be a Carathéodory map such that (4.1) holds true. Let $F: I \times H \rightrightarrows H$ be a set-valued map with non-empty convex weakly compact values satisfying $(j)-(j j)-(j j j)$. Then, for any $x_{0} \in \operatorname{dom} \varphi\left(T_{0}, \cdot\right)$, there exists an absolutely continuous solution $x(\cdot)$ of the differential inclusion

$$
\left\{\begin{array}{l}
-\dot{x}(t) \in \partial \varphi(t, x(t))+F(t, x(t))+f(t, x(t)) \quad \text { a.e. } t \in I, \\
x\left(T_{0}\right)=x_{0} .
\end{array}\right.
$$

More precisely, there exists an absolutely continuous map $x(\cdot): I \rightarrow H$ and an integrable map $g(\cdot): I \rightarrow H$ such that $x\left(T_{0}\right)=x_{0}, x(t) \in \operatorname{dom} \varphi(t, \cdot)$ for all $t \in I$, and for almost all $t \in I, g(t) \in$ $F(t, x(t))$ and $-\dot{x}(t)-g(t)-f(t, x(t)) \in \partial \varphi(t, x(t))$, with

$$
\|g(t)\| \leq \beta(t)(1+\|x(t)\|) .
$$

Moreover, the following inequalities hold true

$$
\int_{T_{0}}^{T}\|\dot{x}(t)\|^{2} d t \leq d_{*}+2 \sigma \int_{T_{0}}^{T}\|g(t)\|^{2} d t+2 \sigma \int_{T_{0}}^{T}\|f(t, x(t))\|^{2} d t,
$$

and

$$
\int_{T_{0}}^{T}\|\dot{x}(t)\|^{2} d t \leq d_{*}+4 \sigma \int_{T_{0}}^{T}\left(\beta^{2}(s)+\alpha^{2}(s)\right)\left(1+\|x(s)\|^{2}\right) d s
$$


where

$$
\begin{aligned}
d_{*}= & \left(k^{2}(0)+3(\rho+1)^{2}\right) \int_{T_{0}}^{T} \dot{a}^{2}(t) d t+2\left[T-T_{0}+\varphi\left(T_{0}, x_{0}\right)-\varphi(T, x(T))\right], \\
& \sigma=k^{2}(0)+3(\rho+1)^{2}+4 .
\end{aligned}
$$

Proof. In the proof, we use a discretisation approach. Define, for any $(t, x) \in I \times H$, the element of minimal norm of $F(t, x)$ by $h(t, x)=P_{F(t, x)}(0)$, where $P_{F(t, x)}(0)$ denotes the projection of the origin onto $F(t, x)$. Thanks to the result of [33], for any $x \in H$, the map $t \mapsto h(t, x)$ is $\mathscr{L}(I)$-measurable and satisfies (by $(j j j)$ )

$$
h(t, x) \in F(t, x) \text { and }\|h(t, x)\| \leq \beta(t)(1+\|x\|) \text { for all }(t, x) \in I \times H .
$$

Let us consider

Suppose that

$$
m=\frac{1}{8\left(T-T_{0}\right)\left[k^{2}(0)+3(\rho+1)^{2}+4\right]}>0 .
$$

$$
\int_{T_{0}}^{T}\left[\beta^{2}(s)+\alpha^{2}(s)\right] d s<m
$$

(I) Let us construct the sequence $\left(x_{n}\right)$.

For any $n \in \mathbb{N}^{*}$, define a partition of $I:=\left[T_{0}, T\right]$ with

$$
t_{i}^{n}=T_{0}+i \frac{T-T_{0}}{n}(0 \leq i \leq n) .
$$

Then, consider the following problem

$$
\left\{\begin{aligned}
-\dot{x}(t) & \in \partial \varphi(t, x(t))+f\left(t, x_{0}\right)+h\left(t, x_{0}\right) \quad \text { a.e. } t \in\left[t_{0}^{n}, t_{1}^{n}\right] \\
x\left(t_{0}^{n}\right) & =x_{0} \in \operatorname{dom} \varphi\left(t_{0}^{n}, \cdot\right) .
\end{aligned}\right.
$$

In view of Proposition 3.2, there exists $x^{n, 0}(\cdot):\left[t_{0}^{n}, t_{1}^{n}\right] \rightarrow H$, an absolutely continuous solution of the problem above (since by condition (4.1) and (4.6) $f\left(\cdot, x_{0}\right)+h\left(\cdot, x_{0}\right) \in L_{H}^{2}\left(\left[t_{0}^{n}, t_{1}^{n}\right]\right)$ ) such that $x^{n, 0}(t) \in \operatorname{dom} \varphi(t, \cdot)$ for all $t \in\left[t_{0}^{n}, t_{1}^{n}\right]$. Put $x_{0}^{n}=x_{0}, x_{1}^{n}=x^{n, 0}\left(t_{1}^{n}\right)$. Thanks to (3.2), this solution satisfies

where

$$
\int_{t_{0}^{n}}^{t_{1}^{n}}\left\|\dot{x}^{n, 0}(t)\right\|^{2} d t \leq \sigma \int_{t_{0}^{n}}^{t_{1}^{n}}\left\|f\left(t, x_{0}^{n}\right)+h\left(t, x_{0}^{n}\right)\right\|^{2} d t+d_{0},
$$

$$
\begin{aligned}
\sigma & =k^{2}(0)+3(\rho+1)^{2}+4 \\
d_{0} & =\left[k^{2}(0)+3(\rho+1)^{2}\right] \int_{t_{0}^{n}}^{t_{1}^{n}} \dot{a}^{2}(t) d t+2\left[\left(t_{1}^{n}-t_{0}^{n}\right)+\varphi\left(t_{0}^{n}, x_{0}^{n}\right)-\varphi\left(t_{1}^{n}, x_{1}^{n}\right)\right] .
\end{aligned}
$$

In a similar way, the differential inclusion

$$
\left\{\begin{aligned}
-\dot{x}(t) & \in \partial \varphi(t, x(t))+f\left(t, x_{1}^{n}\right)+h\left(t, x_{1}^{n}\right) \quad \text { a.e. } t \in\left[t_{1}^{n}, t_{2}^{n}\right] \\
x\left(t_{1}^{n}\right) & =x_{1}^{n} \in \operatorname{dom} \varphi\left(t_{1}^{n}, \cdot\right)
\end{aligned}\right.
$$

admits an absolutely continuous solution $x^{n, 1}(\cdot):\left[t_{1}^{n}, t_{2}^{n}\right] \rightarrow H$ with $x^{n, 1}\left(t_{1}^{n}\right)=x^{n, 0}\left(t_{1}^{n}\right)$ and satisfying (3.2) on $\left[t_{1}^{n}, t_{2}^{n}\right]$. In a similar way, for each $i \in\{2, \cdots, n-1\}$, putting $x_{i}^{n}=x^{n, i-1}\left(t_{i}^{n}\right)$, we see that the differential inclusion

$$
\left\{\begin{aligned}
-\dot{x}(t) & \in \partial \varphi(t, x(t))+f\left(t, x_{i}^{n}\right)+h\left(t, x_{i}^{n}\right) \quad \text { a.e. } t \in\left[t_{i}^{n}, t_{i+1}^{n}\right] \\
x\left(t_{i}^{n}\right) & =x_{i}^{n} \in \operatorname{dom} \varphi\left(t_{i}^{n}, \cdot\right)
\end{aligned}\right.
$$


admits an absolutely continuous solution $x^{n, i}(\cdot):\left[t_{i}^{n}, t_{i+1}^{n}\right] \rightarrow H$ such that $x^{n, i}(t) \in \operatorname{dom} \varphi(t, \cdot)$ for all $t \in\left[t_{i}^{n}, t_{i+1}^{n}\right]$ with $x^{n, i}\left(t_{i}^{n}\right)=x^{n, i-1}\left(t_{i}^{n}\right)$ and satisfying (3.2) on $\left[t_{i}^{n}, t_{i+1}^{n}\right]$. Consequently, for any $i \in\{0, \cdots, n-1\}$ one has (by (3.2))

$$
\int_{t_{i}^{n}}^{t_{i+1}^{n}}\left\|\dot{x}^{n, i}(t)\right\|^{2} d t \leq \sigma \int_{t_{i}^{n}}^{t_{i+1}^{n}}\left\|f\left(t, x_{i}^{n}\right)+h\left(t, x_{i}^{n}\right)\right\|^{2} d t+d_{i},
$$

where

$$
d_{i}=\left[k^{2}(0)+3(\rho+1)^{2}\right] \int_{t_{i}^{n}}^{t_{i+1}^{n}} \dot{a}^{2}(t) d t+2\left[\left(t_{i+1}^{n}-t_{i}^{n}\right)+\varphi\left(t_{i}^{n}, x_{i}^{n}\right)-\varphi\left(t_{i+1}^{n}, x_{i+1}^{n}\right)\right] .
$$

By (4.1), (4.6), for all $0 \leq i \leq n-1$, this estimate may be written as follows

$$
\int_{t_{i}^{n}}^{t_{i+1}^{n}}\left\|\dot{x}^{n, i}(t)\right\|^{2} d t \leq 4 \sigma \int_{t_{i}^{n}}^{t_{i+1}^{n}}\left(\alpha^{2}(t)+\beta^{2}(t)\right)\left(1+\left\|x_{i}^{n}\right\|^{2}\right) d t+d_{i} .
$$

Define $x_{n}:\left[T_{0}, T\right] \rightarrow H$ by

$$
x_{n}(t)=x^{n, i}(t) \forall t \in\left[t_{i}^{n}, t_{i+1}^{n}\right], i \in\{0, \cdots, n-1\} .
$$

Obviously $x_{n}(\cdot)$ is absolutely continuous on $\left[T_{0}, T\right]$, and putting

$$
\left.\left\{\begin{array}{l}
\theta_{n}\left(T_{0}\right)=T_{0}, \\
\theta_{n}(t)=t_{i}^{n}
\end{array} \quad \text { if } t \in\right] t_{i}^{n}, t_{i+1}^{n}\right], i \in\{0, \cdots, n-1\},
$$

one has

$$
\left\{\begin{array}{l}
-\dot{x}_{n}(t) \in \partial \varphi\left(t, x_{n}(t)\right)+f\left(t, x_{n}\left(\theta_{n}(t)\right)\right)+h\left(t, x_{n}\left(\theta_{n}(t)\right)\right) \text { a.e. } t \in I, \\
x_{n}\left(T_{0}\right)=x_{0} .
\end{array}\right.
$$

Set

$$
f_{n}(t)=f\left(t, x_{n}\left(\theta_{n}(t)\right)\right) \text { and } h_{n}(t)=h\left(t, x_{n}\left(\theta_{n}(t)\right)\right), \forall t \in\left[T_{0}, T\right] .
$$

In view of (4.10), for any $i \in\{0, \cdots, n-1\}$, one has

$$
\begin{aligned}
\int_{t_{i}^{n}}^{t_{i+1}^{n}}\left\|\dot{x}_{n}(t)\right\|^{2} d t & \leq 4 \sigma\left(1+\left\|x_{n}\left(t_{i}^{n}\right)\right\|^{2}\right) \int_{t_{i}^{n}}^{t_{i+1}^{n}}\left(\alpha^{2}(t)+\beta^{2}(t)\right) d t+d_{i} \\
& \leq 4 \sigma\left(1+\max _{0 \leq i \leq n}\left\|x_{n}\left(t_{i}^{n}\right)\right\|^{2}\right) \int_{t_{i}^{n}}^{t_{i+1}^{n}}\left(\alpha^{2}(t)+\beta^{2}(t)\right) d t+d_{i} .
\end{aligned}
$$

For any $i \in\{0, \cdots, n-1\}$, one has

$$
\int_{T_{0}}^{T}\left\|\dot{x}_{n}(t)\right\|^{2} d t \leq 4 \sigma\left(1+\left\|x_{n}(\cdot)\right\|_{\infty}^{2}\right) \int_{T_{0}}^{T}\left(\beta^{2}(t)+\alpha^{2}(t)\right) d t+\sum_{i=0}^{n-1} d_{i}
$$

where

$$
d_{n}^{\prime}=\sum_{i=0}^{n-1} d_{i}=\left[k^{2}(0)+3(\rho+1)^{2}\right] \int_{T_{0}}^{T} \dot{a}^{2}(t) d t+2\left[T-T_{0}+\varphi\left(T_{0}, x_{0}\right)-\varphi\left(T, x_{n}(T)\right)\right] .
$$

As the function $\varphi$ takes positive real values, one has $-\varphi\left(T, x_{n}(T)\right) \leq 0$. Then,

$$
\sum_{i=0}^{n-1} d_{i} \leq\left[k^{2}(0)+3(\rho+1)^{2}\right] \int_{T_{0}}^{T} \dot{a}^{2}(t) d t+2\left[\left(T-T_{0}\right)+\varphi\left(T_{0}, x_{0}\right)\right]=d .
$$


As a consequence,

$$
\int_{T_{0}}^{T}\left\|\dot{x}_{n}(t)\right\|^{2} d t \leq b+l\left\|x_{n}(\cdot)\right\|_{\infty}^{2}
$$

where

$$
b=4 \sigma \int_{T_{0}}^{T}\left(\alpha^{2}(t)+\beta^{2}(t)\right) d t+d \quad \text { and } \quad l=4 \sigma \int_{T_{0}}^{T}\left(\alpha^{2}(t)+\beta^{2}(t)\right) d t .
$$

Using the Cauchy-Schwarz inequality and (4.13), one has, for all $s \in I$,

$$
\left\|x_{n}(s)-x_{0}\right\|^{2} \leq\left(s-T_{0}\right)\left(\int_{T_{0}}^{s}\left\|\dot{x}_{n}(t)\right\|^{2} d t\right) \leq\left(T-T_{0}\right)\left(b+l\left\|x_{n}(\cdot)\right\|_{\infty}^{2}\right)
$$

and hence

$$
\left\|x_{n}(s)\right\|^{2} \leq 2\left\|x_{0}\right\|^{2}+2\left\|x_{n}(s)-x_{0}\right\|^{2} \leq 2\left\|x_{0}\right\|^{2}+2\left(T-T_{0}\right)\left(b+l\left\|x_{n}(\cdot)\right\|_{\infty}^{2}\right) .
$$

Consequently, for each $n$, one gets

$$
\left(1-2\left(T-T_{0}\right) l\right)\left\|x_{n}(\cdot)\right\|_{\infty}^{2} \leq 2\left(\left\|x_{0}\right\|^{2}+\left(T-T_{0}\right) b\right) .
$$

According to (4.7), that is, $2\left(T-T_{0}\right) l<1$, one has, for any $t$ and for any integer $n$,

$$
\left\|x_{n}(\cdot)\right\|_{\infty} \leq M
$$

and

$$
\begin{gathered}
\left\|f_{n}(t)\right\|=\left\|f\left(t, x_{n}\left(\theta_{n}(t)\right)\right)\right\| \leq \alpha(t)(1+M), \\
\left\|h_{n}(t)\right\|=\left\|h\left(t, x_{n}\left(\theta_{n}(t)\right)\right)\right\| \leq \beta(t)(1+M),
\end{gathered}
$$

where

$$
M:=\left(\frac{2\left(\left\|x_{0}\right\|^{2}+\left(T-T_{0}\right) b\right)}{1-2\left(T-T_{0}\right) l}\right)^{\frac{1}{2}} .
$$

In a straight way, (4.13) and (4.14) yield

$$
\sup _{n \in \mathbb{N}} \int_{T_{0}}^{T}\left\|\dot{x}_{n}(t)\right\|^{2} d t \leq b+l M^{2}:=M_{1} .
$$

As a result, in view of (4.15), (4.16) and (4.17), one deduces that

$$
\sup _{n \in \mathbb{N}}\left\|\dot{x}_{n}(\cdot)+f\left(\cdot, x_{n}\left(\theta_{n}(\cdot)\right)\right)+h\left(\cdot, x_{n}\left(\theta_{n}(\cdot)\right)\right)\right\|_{L_{H}^{2}(I)}<+\infty .
$$

(II) Let us prove the convergence of the sequence $\left(x_{n}(\cdot)\right)$.

In view of (4.14), we have that $\left(x_{n}(\cdot)\right)$ is bounded. As $x_{n}(t) \in \operatorname{dom} \varphi(t, \cdot)$ for all $t \in I$ and the assumption that $\operatorname{dom} \varphi(t, \cdot)$ is ball-compact for every $t \in I$, it results that the set $\left\{x_{n}(t)\right\}$ is relatively compact for the norm topology of $H$. Moreover,

$$
\begin{aligned}
\left\|x_{n}(t)-x_{n}(s)\right\| & =\left\|\int_{s}^{t} \dot{x}_{n}(\tau) d \tau\right\| \\
& \leq(t-s)^{1 / 2}\left(\int_{T_{0}}^{T}\left\|\dot{x}_{n}(\tau)\right\|^{2} d \tau\right)^{1 / 2} \\
& \leq(t-s)^{1 / 2} M_{1}^{1 / 2}
\end{aligned}
$$


that is, $\left(x_{n}(\cdot)\right)$ is equicontinuous. Thus, by Ascoli's theorem, $\left.\left(x_{n}(\cdot)\right)\right)$ is relatively compact in $\mathscr{C}_{H}(I)$. So, we can extract a subsequence of $\left(x_{n}(\cdot)\right)_{n}$ that uniformly converges on $I$ to some map $x(\cdot) \in \mathscr{C}_{H}(I)$ satisfying $x\left(T_{0}\right)=x_{0}$. Further, From

$$
\begin{aligned}
\left\|x_{n}\left(\theta_{n}(t)\right)-x(t)\right\| & \leq\left\|x_{n}\left(\theta_{n}(t)\right)-x_{n}(t)\right\|+\left\|x_{n}(t)-x(t)\right\| \\
& \leq\left(t-\theta_{n}(t)\right)^{\frac{1}{2}} M_{1}^{\frac{1}{2}}+\left\|x_{n}(t)-x(t)\right\|,
\end{aligned}
$$

and the construction $0 \leq t-\theta_{n}(t) \leq \frac{T-T_{0}}{n}$ for any $t \in I$, we deduce that

$$
\lim _{n \rightarrow \infty}\left\|x_{n}\left(\theta_{n}(t)\right)-x(t)\right\|=0 \text { for any } t \in I .
$$

In view of (4.17), up to a subsequence that we do not relabel, $\left(\dot{x}_{n}(\cdot)\right)$ weakly converges in $L_{H}^{2}(I)$ to some element $z(\cdot)$. Relying on the absolute continuity of $\left(x_{n}(\cdot)\right)_{n}$, for any integer $n$ and any $e \in H$ and for $T_{0} \leq s \leq t \leq T$, we can write

$$
\begin{aligned}
\left\langle e, \int_{s}^{t} \dot{x}_{n}(\tau) d \tau\right\rangle & =\int_{T_{0}}^{T}\left\langle e \mathbf{1}_{] s, t]}(\tau), \dot{x}_{n}(\tau)\right\rangle d \tau \\
& =\left\langle e, x_{n}(t)-x_{n}(s)\right\rangle,
\end{aligned}
$$

where $\mathbf{1}_{] s, t]}$ denotes the characteristic function of the interval $\left.] s, t\right]$. Next, passing to the limit in the equality yields

$$
\left\langle e, \int_{s}^{t} z(\tau) d \tau\right\rangle=\langle e, x(t)-x(s)\rangle .
$$

Therefore, given any $s, t \in I$ with $s \leq t$, we get $\int_{s}^{t} z(\tau) d \tau=x(t)-x(s)$, and hence $x(\cdot)$ is absolutely continuous and $z(\cdot)$ coincides almost everywhere in $I$ with $\dot{x}(\cdot)$. Thus, $\dot{x} \in L_{H}^{2}(I)$ and

$$
\dot{x}_{n} \rightarrow \dot{x} \quad \text { weakly in } L_{H}^{2}(I) \text {. }
$$

(III) Let us prove

$$
-\dot{x}(t) \in \partial \varphi(t, x(t))+f(t, x(t))+F(t, x(t)) \quad \text { a.e. } t \in I .
$$

By (4.16), one gets

$$
\sup _{n} \int_{T_{0}}^{T}\left\|h_{n}(s)\right\|^{2} d s \leq(1+M)^{2} \int_{T_{0}}^{T} \beta^{2}(t) d t<+\infty .
$$

Hence, up to a subsequence still denoted $\left(h_{n}\right)$, there exists some map $g$ such that

$$
h_{n} \rightarrow g \text { weakly in } L_{H}^{2}(I) \text {. }
$$

Thanks to (4.19) and the continuity of $f$ with respect to its second variable, one has, for a.e. $t \in I$,

$$
\lim _{n \rightarrow \infty}\left\|f\left(t, x_{n}\left(\theta_{n}(t)\right)\right)-f(t, x(t))\right\|=0,
$$

which together with (4.15) implies that

$$
\lim _{n \rightarrow \infty} \int_{T_{0}}^{T}\left\|f\left(t, x_{n}\left(\theta_{n}(t)\right)\right)-f(t, x(t))\right\|^{2} d t=0 .
$$

In view of the convergence obtained in (4.20) (4.21) and (4.22) (which entails weak convergence in $\left.L_{H}^{2}(I)\right)$ and the strong convergence of $\left(x_{n}(\cdot)\right)$ in $L_{H}^{2}(I)$ to $x(\cdot)$, we conclude from Proposition 3.1 that

$$
-\dot{x}(t) \in \partial \varphi(t, x(t))+f(t, x(t))+g(t) \quad \text { a.e. } t \in I .
$$


It remains to show that

$$
g(t) \in F(t, x(t)) \text { a.e } t \in I .
$$

By construction (see (4.6)), we have that $h_{n}(t) \in F\left(t, x_{n}\left(\theta_{n}(t)\right)\right)$ for all $t \in I$ and all $n \geq 1$. Recall that $\left(x_{n}\left(\theta_{n}(t)\right)\right)$ pointwise converges to $x(t)$ for all $t \in I$ and $\left(h_{n}\right)_{n}$ weakly converges in $L_{H}^{2}(I)$ to $g$. This yields, for each $E \in \mathscr{L}\left(\left[T_{0}, T\right]\right)$ and for each $e \in H$,

$$
\begin{aligned}
\int_{E}\langle e, g(t)\rangle d t=\lim _{n} \int_{E}\left\langle e, h_{n}(t)\right\rangle d t & \leq \limsup _{n} \int_{E} \delta^{*}\left(e, F\left(t, x_{n}\left(\theta_{n}(t)\right)\right)\right) d t \\
& \leq \int_{E} \limsup _{n} \delta^{*}\left(e, F\left(t, x_{n}\left(\theta_{n}(t)\right)\right)\right) d t \\
& \leq \int_{E} \delta^{*}(e, F(t, x(t))) d t .
\end{aligned}
$$

Consequently,

$$
\langle e, g(t)\rangle \leq \delta^{*}(e, F(t, x(t))) \text {, a.e. } t \in\left[T_{0}, T\right] .
$$

From the separability of $H$ and [33, Proposition III.35], one gets

$$
g(t) \in F(t, x(t)) \text {, a.e. } t \in\left[T_{0}, T\right] .
$$

Since (4.8) holds for $i=0, \cdots, n-1$, then it follows

$$
\int_{T_{0}}^{T}\left\|\dot{x}_{n}(t)\right\|^{2} d t \leq \sigma \int_{T_{0}}^{T}\left\|f_{n}(t)+h_{n}(t)\right\|^{2} d t+d_{n}^{\prime},
$$

where $d_{n}^{\prime}$ is defined by (4.11). As a consequence, one gets the following useful estimates

$$
\int_{T_{0}}^{T}\left\|\dot{x}_{n}(t)\right\|^{2} d t \leq 2 \sigma \int_{T_{0}}^{T}\left\|f_{n}(t)\right\|^{2} d t+2 \sigma \int_{T_{0}}^{T}\left\|h_{n}(t)\right\|^{2} d t+d_{n}^{\prime}
$$

and

$$
\int_{T_{0}}^{T}\left\|\dot{x}_{n}(t)\right\|^{2} d t \leq 4 \sigma \int_{T_{0}}^{T}\left(\beta^{2}(t)+\alpha^{2}(t)\right)\left(1+\left\|x_{n}\left(\theta_{n}(t)\right)\right\|^{2}\right) d t+d_{n}^{\prime}
$$

Then, passing to the limit as $n$ tends to $\infty$ in (4.24), and using (4.20)-(4.21)-(4.22) yield (4.2). Using (4.19)-(4.20), and passing to the limit as $n$ tends to $\infty$ in (4.25), we have that estimate (4.3) holds for real positive constants $\sigma$ and $d_{*}$ given by (4.4) and (4.5).

To end the proof, we address the general case when $\int_{T_{0}}^{T}\left(\alpha^{2}(s)+\beta^{2}(s)\right) d s \geq m$. Fixing some $\xi>0$ such that for each subinterval $J$ of $I$ with length $(J)<\xi$, one has $\int_{J}\left(\beta^{2}(s)+\alpha^{2}(s)\right) d s<m$. Fix some integer $N$ such that $\left(T-T_{0}\right) / N<\xi$ and put $T_{i}:=T_{0}+\frac{i}{N}\left(T-T_{0}\right)$ for $i=0, \cdots, N$. Then, for each $i=1, \cdots, N$,

$$
\int_{T_{i-1}}^{T_{i}}\left(\alpha^{2}(s)+\beta^{2}(s)\right) d s<m<\frac{1}{8\left(T_{i}-T_{i-1}\right)\left(k^{2}(0)+3(\rho+1)^{2}+4\right)},
$$

and (4.7) relative to the interval $\left[T_{i-1}, T_{i}\right]$ is fulfilled. As a result, we may apply what precedes to the intervals $\left[T_{0}, T_{1}\right],\left[T_{1}, T_{2}\right], \cdots$, and $\left[T_{N-1}, T\right]$ to get absolutely continuous solutions $y_{1}(\cdot)$ on $\left[T_{0}, T_{1}\right]$ with $y_{1}\left(T_{0}\right)=x_{0}, y_{2}(\cdot)$ on $\left[T_{1}, T_{2}\right]$ with $y_{2}\left(T_{1}\right)=y_{1}\left(T_{1}\right), \cdots, y_{N}(\cdot)$ on $\left[T_{N-1}, T\right]$ with $y_{N}\left(T_{N-1}\right)=y_{N-1}\left(T_{N-1}\right)$. Clearly, the map $x(\cdot)$ from $I:=\left[T_{0}, T\right]$ into $H$ defined by $x(t)=y_{i}(t)$ for all $t \in\left[T_{i-1}, T_{i}\right], i=1,2, \cdots, N$ is an absolutely continuous solution on $I$ to the considered problem. Moreover, estimates (4.2)-(4.3) are satisfied on the whole interval. The proof is then complete.

We next present the following proposition. 
Proposition 4.1. Let us use the notation of Theorem 4.1. Then, any absolutely continuous solution $x(\cdot)$ of our problem satisfies

$$
\|f(t, x(t))\| \leq \alpha(t)(1+L) \quad \text { and } \quad\|g(t)\| \leq \beta(t)(1+L) \text { a.e. } t \in I,
$$

where

$$
\|x(\cdot)\|_{\infty} \leq L
$$

with

$$
L:=\left\|x_{0}\right\|+[\xi(T)]^{\frac{1}{2}},
$$

where $\xi(\cdot)$ is the increasing, continuous, and non-negative function defined on $\left[T_{0}, T\right]$ by

$$
\xi(s)=b(s)+4 \sigma\left(s-T_{0}\right) \int_{T_{0}}^{s} b(\tau)\left[\beta^{2}(\tau)+\alpha^{2}(\tau)\right] \exp \left(4 \sigma \int_{\tau}^{s} \theta\left[\beta^{2}(\theta)+\alpha^{2}(\theta)\right] d \theta\right) d \tau,
$$

and, for each $t \in\left[T_{0}, T\right]$,

$$
b(t)=\left(t-T_{0}\right)\left(d_{*}+4 \sigma\left(1+\left\|x_{0}\right\|\right)^{2} \int_{T_{0}}^{t}\left[\beta^{2}(\tau)+\alpha^{2}(\tau)\right] d \tau\right) .
$$

Remark 4.1. (1) Assumption $(j j j)$ is weaker than the one used in Theorem 2 [12]. $(j j j)^{\prime}$ for some $\beta \in L_{\mathbb{R}_{+}}^{2}(I), F$ satisfies the following condition

$$
F(t, x) \subset \beta(t)(1+\|x\|) \bar{B}_{H} \text { for all }(t, x) \in I \times H .
$$

(2) It is worth pointing out that the case when $f=0$ and $F$ satisfies $(j)-(j j)-(j j j)^{\prime}$ has been established (see Theorem 2 [12]) based on Kakutani-Ky Fan fixed point theorem. Following the arguments used there, we extend the existence result to the case $F+f$ only under restrictive conditions on $I, f$ and $\beta$.

(3) On the perturbation $f, F=0$, let us mention that, in [19], $f$ is assumed to be in addition Lipschitz with respect to its second variable. This assumption is crucial in order to get the uniqueness of the solution [19]. In the case that we replace the ball-compactness assumption on dom $\varphi(t, \cdot)$ by a compactness assumption on $F$ when $f(\cdot, \cdot)$ is Lipschitz in $x$, the perturbed problem by $f+F$ can be treated using the Cauchy criterion as in [19].

We derive from Theorem 4.1 the special case of the sweeping process, that is, $\varphi(t, x)=$ $\psi_{C(t)}(x)$, where $\psi_{C(t)}$ is the indicator function of a subset $C(t)$ of $H$ for all $(t, x) \in I \times H$.

Corollary 4.1. Let $H$ be a real separable Hilbert space. Let $C: I \rightrightarrows H$ be a set-valued map satisfying the $\left(H_{1}^{\prime}\right)-\left(H_{2}^{\prime}\right)$ of Remark 3.1. Moreover, assume that $C(t)$ is ball-compact for all $t \in I$. Let $f$ (resp. $F$ ) be a single-valued (resp. set-valued) map satisfying the assumptions of Theorem 4.1. Then, the problem

$$
\left\{\begin{array}{l}
-\dot{x}(t) \in N_{C(t)}(x(t))+F(t, x(t))+f(t, x(t)) \quad \text { a.e. } t \in I \\
x_{0}=x\left(T_{0}\right) \in C\left(T_{0}\right)
\end{array}\right.
$$

admits an absolutely continuous solution.

Now, let us recall the concept of $r$-uniformly lower semicontinuous from the right introduced by Tolstonogov [35]. 
Definition 4.1. The set-valued map $C: I \rightrightarrows H$ with nonempty convex closed values is said to be $r$-uniformly lower semicontinuous from the right if there exists a family $\mathscr{B}=\left\{a_{r}, r \geq 0\right\} \subset$ $W^{1, p}(I, \mathbb{R})$ such that for any $r \geq 0$ and any $s, t \in I s \leq t,\|x\| \leq r$, the inequality

$$
d(x, C(t)) \leq d(x, C(s))+\left|a_{r}(t)-a_{r}(s)\right|,
$$

holds.

We end this section by the following remak.

Remark 4.2. The class of the sweeping process where the moving sets are $r$-uniformly lower semicontinuous from the right is more general than the one involving the $\left(H_{2}^{\prime}\right)$ in Corollary 4.1. Indeed, let $C: I \rightrightarrows H$ be a set-valued map satisfying the $\left(H_{1}^{\prime}\right)-\left(H_{2}^{\prime}\right)$ of Remark 3.1. From [36], one has

$$
d_{H}(C(t), C(s))=\sup _{x \in H}\{|d(x, C(t))-d(x, C(s))|\},
$$

which together with $\left(H_{2}^{\prime}\right)$ yields that there exists $a \in W^{1,2}(I, \mathbb{R})$ such that

$$
|d(x, C(t))-d(x, C(s))| \leq|a(t)-a(s)| \forall t, s \in I, \forall x \in H .
$$

So, there exists a family $\mathscr{B}=\left\{a_{r}, r \geq 0\right\} \subset W^{1, p}(I, \mathbb{R})\left(a_{r}=a, p=2\right)$ such that, for any $r \geq 0$ and any $s, t \in I s \leq t,\|x\| \leq r$, the inequality

$$
d(x, C(t)) \leq d(x, C(s))+\left|a_{r}(t)-a_{r}(s)\right|,
$$

holds.

\section{THE EVOLUTION INCLUSION INVOLVING FRACTIONAL DIFFERENTIAL EQUATIONS}

The differential equations of fractional order have recently been proved valuable tools in modelling many phenomena in various fields of science and engineering. There are numerous applications to problems in viscoelasticity, electrochemistry, control, porous media, electromagnetics, etc. There has been a significant theoretical development in fractional differential equations in recent years; see, for instance, [37, 38], and the references therein.

More recently, considerable attention has been given to the existence of solutions of boundary value problems and boundary conditions for implicit fractional differential equations and integral equations with the Caputo fractional derivative. We refer the reader to, e.g., [39, 40], and the references therein.

The aim of this section is to connect differential inclusions with the Caputo fractional derivatives in the context of finite dimensional setting. This section is dedicated to the differential inclusion coupled by a fractional differential equation based on the result obtained in Theorem 4.1 .

In the sequel, let $H=\mathbb{R}^{d}$. To begin with, let us recall some needed background material in fractional calculus (see [37, 39, 41]).

Let $f \in L_{H}^{1}([0, T])$ and give the following definitions and properties.

Definition 5.1. The Riemann-Liouville fractional integral of order $\alpha>0$ of the function $f$ is defined by

$$
I^{\alpha} f(t):=\frac{1}{\Gamma(\alpha)} \int_{0}^{t}(t-s)^{\alpha-1} f(s) d s, t \in[0, T] .
$$


If $f \in L_{H}^{\infty}([0, T])$ and $\alpha>0$, then $I^{\alpha} f:[0, T] \rightarrow H$ is continuous, that is, $I^{\alpha} f \in \mathscr{C}_{H}([0, T])$. The Riemann-Liouville fractional derivative of order $\alpha>0$ of the function $f$ is defined by

$$
\left(D^{\alpha} f\right)(t):=\frac{1}{\Gamma(n-\alpha)}\left(\frac{d}{d t}\right)^{n} \int_{0}^{t}(t-s)^{n-\alpha-1} f(s) d s, t \in[0, T],
$$

where $n=[\alpha]+1$ and $[\alpha]$ denotes the integer part of $\alpha$.

The Riemann-Liouville fractional derivatives are linear. If $0<\beta<\alpha$, then, for $f \in L_{H}^{1}([0, T])$,

$$
\left(D^{\beta} I^{\alpha} f\right)(t)=I^{\alpha-\beta} f(t) .
$$

Definition 5.2. The Caputo fractional derivative of order $\alpha>0$ of the function $f$ is defined by

$$
\left({ }^{c} D^{\alpha} f\right)(t)=\frac{1}{\Gamma(n-\alpha)} \int_{0}^{t} \frac{f^{(n)}(s)}{(t-s)^{1-n+\alpha}} d s,
$$

where $n=[\alpha]+1$.

The Caputo fractional derivatives are linear. The Caputo fractional derivative of a constant is equal to zero. If $\alpha \notin \mathbb{N}$, then the Caputo fractional derivatives coincide with the RiemannLiouville fractional derivatives

$$
\left({ }^{c} D^{\alpha} f\right)(t)=D^{\alpha} f(t),
$$

if $f(0)=f^{\prime}(0)=\cdots=f^{(n-1)}(0)=0$. Let $y \in L_{H}^{\infty}([0, T])$ or $y \in \mathscr{C}_{H}([0, T])$. Then,

$$
\left({ }^{c} D^{\alpha} I^{\alpha} f\right)(t)=f(t), t \in[0, T] .
$$

Denote by

$$
\mathscr{C}_{H}^{1}([0, T])=\left\{u \in \mathscr{C}_{H}([0, T]): \frac{d u}{d t} \in \mathscr{C}_{H}([0, T])\right\},
$$

where $\frac{d u}{d t}$ is the derivative of $u$,

$$
W_{H}^{\alpha, \infty}([0, T])=\left\{u \in \mathscr{C}_{H}^{1}([0, T]):{ }^{c} D^{\alpha-1} u \in \mathscr{C}_{H}([0, T]) ;{ }^{c} D^{\alpha} u \in L_{H}^{\infty}([0, T])\right\},
$$

where ${ }^{c} D^{\alpha-1} u$ and ${ }^{c} D^{\alpha} u$ are the fractional Caputo derivatives of order $\alpha-1$ and $\alpha$ of $u$, respectively.

Next, let $\alpha \in] 1,2]$. Recall the following lemma (see [40]).

Lemma 5.1. Let $f \in L_{H}^{\infty}([0, T])$ and $x \in H$. Then, the function $u:[0, T] \rightarrow H$ is a $W_{H}^{\alpha, \infty}([0, T])$ solution to the fractional differential equation

$$
\left\{\begin{array}{l}
{ }^{c} D^{\alpha} u(t)=f(t), t \in[0, T] \\
u(0)=x, \dot{u}(0)=0
\end{array}\right.
$$

if and only if

$$
u(t)=x+\int_{0}^{t} \frac{(t-s)^{\alpha-1}}{\Gamma(\alpha)} f(s) d s, t \in[0, T] .
$$

Proof. (I) Let $f \in L_{H}^{\infty}([0, T])$ and $u(t)=x+\int_{0}^{t} \frac{(t-s)^{\alpha-1}}{\Gamma(\alpha)} f(s) d s$. Then,

$$
u(t)=x+I^{\alpha} f(t) .
$$

It is clear that $I^{\alpha} f \in \mathscr{C}_{H}([0, T])$ so $u$ is continuous on $[0, T]$. From the expression of $u$, it results

$$
\frac{d u}{d t}(t)=I^{\alpha-1} f(t) .
$$


Hence, it follows from (5.6)-(5.7) that

$$
\begin{aligned}
u(0) & =I^{\alpha} f(0)+x=x, \\
\frac{d u}{d t}(0) & =I^{\alpha-1} f(0)=0 .
\end{aligned}
$$

As a result, one deduces that $u \in \mathscr{C}_{H}^{1}([0, T])$. Now, let us evaluate ${ }^{c} D^{\alpha-1} u$ and ${ }^{c} D^{\alpha} u$. One has

$$
\begin{aligned}
{ }^{c} D^{\alpha-1} u(t) & ={ }^{c} D^{\alpha-1}\left(x+I^{\alpha} f(t)\right) \\
& ={ }^{c} D^{\alpha-1} I^{\alpha} f(t)=D^{\alpha-1} I^{\alpha} f(t) \\
& =I^{\alpha-\alpha+1} f(t)=I^{1} f(t)=\int_{0}^{t} f(s) d s,
\end{aligned}
$$

by using the properties of the Caputo fractional derivative and relations (5.3)-(5.4). From (5.5), one gets

$$
\begin{aligned}
{ }^{c} D^{\alpha} u(t) & ={ }^{c} D^{\alpha} I^{\alpha} f(t) \\
& =f(t)
\end{aligned}
$$

It results that $u \in W_{H}^{\alpha, \infty}([0, T])$.

(II) If $u$ is a $W_{H}^{\alpha, \infty}([0, T])$-solution to

$$
\left({ }^{c} D^{\alpha} u\right)(t)=f(t), u(0)=x, \dot{u}(0)=0,
$$

with $f \in L_{H}^{\infty}([0, T])$, then

$$
u(t)=I^{\alpha} f(t)+x, t \in[0, T]
$$

is a solution to (5.8) by repeating the above computation. Indeed, from (5.9), one has

$$
\left({ }^{c} D^{\alpha} u\right)(t)=f(t), t \in[0, T] \text { and } u(0)=x .
$$

Coming back to the above expression of $I^{\alpha} f$, one gets

$$
u(t)=x+\int_{0}^{t} \frac{(t-s)^{\alpha-1}}{\Gamma(\alpha)} f(s) d s, t \in[0, T]
$$

This ends the proof of Lemma 5.1.

In view of Lemma 5.1, a map $x \in \mathscr{C}_{H}(I)$ possesses a Caputo fractional derivative ${ }^{c} D^{\alpha} x$ on $I$ if and only if there exist $y \in L_{H}^{\infty}(I)$ and $x_{0} \in H$ such that

$$
x(t)=x_{0}+I^{\alpha} y(t) \text { for all } t \in I .
$$

In that case, the above relation holds replacing $y$ by ${ }^{c} D^{\alpha} x$ and $x_{0}$ by $x(0)$.

Now, we are ready to state the main theorem of this section.

Theorem 5.1. Let $\varphi:[0, T] \times H \rightarrow[0,+\infty]$ be a map satisfying $\left(H_{1}\right)-\left(H_{2}\right)$. Let $f:[0, T] \times H \rightarrow H$ be a Carathéodory map such that $\|f(t, x)\| \leq M$ for all $(t, x) \in[0, T] \times H$ and for some positive constant $M$. Let $F: I \times H \rightrightarrows H$ be a set-valued map with non-empty convex compact values satisfying $(j)-(j j)-(j j j)$. Suppose further that there exists a function $\varsigma(\cdot) \in L_{\mathbb{R}_{+}}^{2}([0, T])$ such that $\left\langle y_{1}-y_{2}, x_{1}-x_{2}\right\rangle \geq \varsigma(t)\left\|x_{1}-x_{2}\right\|^{2}$, for all $y_{1} \in F\left(t, x_{1}\right), y_{2} \in F\left(t, x_{2}\right)$ and $t \in[0, T]$. Then, for $\left(u_{0}, x_{0}\right) \in \operatorname{dom} \varphi(0, \cdot) \times H$, there is an absolutely continuous map $u:[0, T] \rightarrow H$, and a $W_{H}^{\alpha, \infty}([0, T])$ map $x:[0, T] \rightarrow H$ satisfying

$$
-\dot{u}(t) \in \partial \varphi(t, u(t))+f(t, x(t))+F(t, u(t)) \text { a.e. } t \in[0, T],
$$




$$
\begin{gathered}
{ }^{c} D^{\alpha} x(t)=u(t), t \in[0, T], \\
u(0)=u_{0} \in \operatorname{dom} \varphi(0, \cdot), \\
x(0)=x_{0} \in H .
\end{gathered}
$$

Proof. We follow some arguments used in [12], but this needs a careful look. In view of Theorem 4.1 , and the assumptions on $f, F$, for any bounded continuous map $h:[0, T] \rightarrow H$, there is an absolutely continuous solution $v_{h}$ to the inclusion

$$
\left(P_{h}\right)\left\{\begin{array}{r}
-\dot{v}_{h}(t) \in \partial \varphi\left(t, v_{h}(t)\right)+f(t, h(t))+F\left(t, v_{h}(t)\right) \text { a.e. } t \in[0, T], \\
v_{h}(0)=u_{0} \in \operatorname{dom} \varphi(0, \cdot),
\end{array}\right.
$$

that is, there exist an absolutely continuous $v_{h}:[0, T] \rightarrow H$ and an $L_{H}^{2}([0, T])$ map $y_{h}:[0, T] \rightarrow H$ such that

$$
\left\{\begin{array}{r}
-\dot{v}_{h}(t) \in \partial \varphi\left(t, v_{h}(t)\right)+f(t, h(t))+y_{h}(t) \text { a.e. } t \in[0, T], \\
v_{h}(0)=u_{0} \in \operatorname{dom} \varphi(0, \cdot),
\end{array}\right.
$$

with $y_{h}(t) \in F\left(t, v_{h}(t)\right)$ a.e. and $\left\|v_{h}(t)\right\| \leq L$ for any $t \in[0, T]$, for some positive constant $L$ (by Proposition 4.1). Moreover, this solution is unique. Indeed, let $v_{1}$ and $v_{2}$ be two solutions of the differential inclusion $\left(P_{h}\right)$ whose existence is guaranteed by Theorem 4.1. Then, there exist two $L_{H}^{2}([0, T])$-functions $y_{1}(\cdot)$ and $y_{2}(\cdot)$ such that, for all $t \in[0, T]$,

$$
y_{1}(t) \in F\left(t, v_{1}(t)\right) \text { and } y_{2}(t) \in F\left(t, v_{2}(t)\right)
$$

and, for almost all $t \in[0, T]$,

$$
-\dot{v}_{1}(t)-y_{1}(t)-f(t, h(t)) \in \partial \varphi\left(t, v_{1}(t)\right),
$$

and

$$
-\dot{v}_{2}(t)-y_{2}(t)-f(t, h(t)) \in \partial \varphi\left(t, v_{2}(t)\right) .
$$

The monotone condition on $F$ yields

$$
\left\langle y_{2}(t)-y_{1}(t), v_{1}(t)-v_{2}(t)\right\rangle \leq-\varsigma(t)\left\|v_{1}(t)-v_{2}(t)\right\|^{2} \leq 0 .
$$

Moreover, the monotone property of $\partial \varphi(t, \cdot)$ ensures that

$$
\left\langle-\dot{v}_{1}(t)-y_{1}(t)-f(t, h(t))-\left(-\dot{v}_{2}(t)-y_{2}(t)-f(t, h(t))\right), v_{1}(t)-v_{2}(t)\right\rangle \geq 0 .
$$

Hence,

$$
\left\langle\dot{v}_{1}(t)-\dot{v}_{2}(t), v_{1}(t)-v_{2}(t)\right\rangle \leq 0
$$

that is,

$$
\frac{1}{2} \frac{d}{d t}\left\|v_{1}(t)-v_{2}(t)\right\|^{2} \leq 0 \text { for a.e. } t \in[0, T] .
$$

Integrating over $[0, T]$ yields $v_{1}=v_{2}$. Then, the solution is unique. Define the closed convex subset $\mathscr{X}$ in the Banach space $\mathscr{C}_{H}([0, T])$ by

$$
\mathscr{X}:=\left\{u_{g}:[0, T] \rightarrow H: u_{g}(t)=x_{0}+\int_{0}^{t} \frac{(t-s)^{\alpha-1}}{\Gamma(\alpha)} g(s) d s, g \in S_{L \bar{B}_{H}}^{1}, t \in[0, T]\right\},
$$

where $S_{L \bar{B}_{H}}^{1}$ denotes the set of all integrable selections of the convex compact valued constant set-valued map $L \bar{B}_{H}$. For each $h \in \mathscr{X}$, consider the map

$$
\Psi(h)(t):=x_{0}+\int_{0}^{t} \frac{(t-s)^{\alpha-1}}{\Gamma(\alpha)} v_{h}(s) d s, t \in[0, T]
$$


where $v_{h}$ denotes the unique absolutely continuous solution to $\left(P_{h}\right)$. Clearly, one has $\Psi(h) \in \mathscr{X}$. For any $h \in \mathscr{X}$ and any $t \in[0, T]$, the inclusion holds

$$
\Psi(h)(t) \in x_{0}+\int_{0}^{T} \frac{(T-s)^{\alpha-1}}{\Gamma(\alpha)} L \bar{B}_{H} d s
$$

As $t \rightrightarrows L \bar{B}_{H}$ is a convex compact valued and integrably bounded (constant) set-valued map, the second member is convex compact valued. It results that, for each $t \in[0, T], \Psi(\mathscr{X})(t)$ is relatively compact. Moreover, the set $\Psi(\mathscr{X})$ is equicontinuous in $\mathscr{C}_{H}([0, T])$. Indeed, let $h \in \mathscr{X}$ and $t, \tau \in[0, T]$ such that $t<\tau$. Then,

$$
\begin{aligned}
& \|\Psi(h)(t)-\Psi(h)(\tau)\| \\
& =\left\|\int_{0}^{t} \frac{(t-s)^{\alpha-1}}{\Gamma(\alpha)} v_{h}(s) d s-\int_{0}^{\tau} \frac{(\tau-s)^{\alpha-1}}{\Gamma(\alpha)} v_{h}(s) d s\right\| \\
& =\left\|\int_{0}^{t} \frac{(t-s)^{\alpha-1}}{\Gamma(\alpha)} v_{h}(s) d s-\int_{0}^{t} \frac{(\tau-s)^{\alpha-1}}{\Gamma(\alpha)} v_{h}(s) d s-\int_{t}^{\tau} \frac{(\tau-s)^{\alpha-1}}{\Gamma(\alpha)} v_{h}(s) d s\right\| \\
& \leq \frac{1}{\Gamma(\alpha)} \int_{0}^{t}\left|(t-s)^{\alpha-1}-(\tau-s)^{\alpha-1}\right|\left\|v_{h}(s)\right\| d s \\
& \quad+\frac{1}{\Gamma(\alpha)} \int_{t}^{\tau}|\tau-s|^{\alpha-1}\left\|v_{h}(s)\right\| d s .
\end{aligned}
$$

Using the inequality $\left|c^{k}-d^{k}\right| \leq|c-d|^{k}$ for all $c, d \geq 0$ and $\left.\left.k \in\right] 0,1\right]$ yields

$$
\|\Psi(h)(t)-\Psi(h)(\tau)\| \leq \frac{L T}{\Gamma(\alpha)}|t-\tau|^{\alpha-1}+\frac{T^{\alpha-1} L}{\Gamma(\alpha)}(\tau-t) .
$$

The last estimate shows that $\Psi(\mathscr{X})$ is equicontinuous in $\mathscr{C}_{H}([0, T])$. The case $\tau<t$ can be treated similarly. By Ascoli's theorem, one deduces that $\Psi(\mathscr{X})$ is relatively compact in the Banach space $\mathscr{C}_{H}([0, T])$. Let us prove that $\Psi$ is continuous. It is sufficient to show that if $\left(h_{n}\right)$ uniformly converges to $h$ in $\mathscr{X}$, then the sequence of absolutely continuous solution $v_{h_{n}}$ associated with $h_{n}$

$$
\left\{\begin{array}{r}
-\dot{v}_{h_{n}}(t) \in \partial \varphi\left(t, v_{h_{n}}(t)\right)+f\left(t, h_{n}(t)\right)+F\left(t, v_{h_{n}}(t)\right) \text { a.e. } \\
v_{h_{n}}(0)=u_{0} \in \operatorname{dom} \varphi(0, \cdot),
\end{array}\right.
$$

uniformly converges to the absolutely continuous solution $v_{h}$ associated with $h$

$$
\left\{\begin{array}{r}
-\dot{v}_{h}(t) \in \partial \varphi\left(t, v_{h}(t)\right)+f(t, h(t))+F\left(t, v_{h}(t)\right) \text { a.e. } \\
v_{h}(0)=u_{0} \in \operatorname{dom} \varphi(0, \cdot) .
\end{array}\right.
$$

For any $n \geq 1$, let $v_{h_{n}}$ be the unique solution of the problem

$$
\left\{\begin{array}{r}
-\dot{v}_{h_{n}}(t) \in \partial \varphi\left(t, v_{h_{n}}(t)\right)+f\left(t, h_{n}(t)\right)+y_{h_{n}}(t), y_{h_{n}}(t) \in F\left(t, v_{h_{n}}(t)\right) \text { a.e. } \\
v_{h_{n}}(0)=u_{0} \in \operatorname{dom} \varphi(0, \cdot),
\end{array}\right.
$$

such that $\left\|v_{h_{n}}(t)\right\| \leq L$. It results that $\left\{v_{h_{n}}(t), h_{n} \in \mathscr{X}\right\}$ is relatively compact in $H$, for any $t \in[0, T]$. Moreover, $\left(v_{h_{n}}\right)$ is equi-absolutely continuous. By Ascoli's theorem, we may assume 
that $\left(v_{h_{n}}\right)$ uniformly converges to an absolutely continuous mapping $z$. By (4.2) in Theorem 4.1, one has

$$
\int_{0}^{T}\left\|\dot{v}_{h_{n}}(t)\right\|^{2} d t \leq d_{*}+2 \sigma \int_{0}^{T}\left(\left\|y_{h_{n}}(t)\right\|^{2}+\left\|f\left(t, h_{n}(t)\right)\right\|^{2}\right) d t .
$$

Define, for any $n \geq 1$, a function $g_{n}$ on $[0, T]$ by

$$
g_{n}(t)=f\left(t, h_{n}(t)\right)+y_{h_{n}}(t), \text { for any } t \in[0, T] .
$$

Then,

$$
\left\|g_{n}(t)\right\| \leq M+\beta(t)(1+L)
$$

for all $t \in[0, T]$. This along with (5.11) yields

$$
\int_{0}^{T}\left\|\dot{v}_{h_{n}}(t)\right\|^{2} d t \leq d_{*}+2 \sigma M^{2} T+2 \sigma(1+L)^{2} \int_{0}^{T} \beta^{2}(t) d t<+\infty .
$$

We may assume that $\left(g_{n}\right)$ weakly converges in $L_{H}^{2}([0, T])$ to some map $g \in L_{H}^{2}([0, T])$, and also that $\left(\dot{v}_{h_{n}}\right)$ weakly converges to some $w \in L_{H}^{2}([0, T])$. Thus, $\left(\dot{v}_{h_{n}}\right)$ weakly converges in $L_{H}^{1}([0, T])$ to $w$ so that

$$
\lim _{n} v_{h_{n}}(t)=u_{0}+\int_{0}^{t} w(s) d s, t \in[0, T]
$$

By identifying the limits, one has

$$
z(t)=u_{0}+\int_{0}^{t} w(s) d s
$$

with $\dot{z}=w$. Moreover, as $f$ is bounded and continuous with respect to its second variable, along with the pointwise convergence of $\left(h_{n}(t)\right)$ to $h(t)(t \in[0, T])$, we find from the dominated convergence theorem that

$$
\lim _{n \rightarrow \infty} \int_{0}^{T}\left\|f\left(t, h_{n}(t)\right)-f(t, h(t))\right\|^{2} d t=0
$$

which entails the weak convergence of $\left(f\left(\cdot, h_{n}(\cdot)\right)\right)$ to $f(\cdot, h(\cdot))$ in $L_{H}^{2}([0, T])$. Since $\left(y_{h_{n}}\right)$ weakly converges in $L_{H}^{2}([0, T])$ to some $y \in L_{H}^{2}([0, T])$ (by $\left.(5.12)\right),\left(v_{h_{n}}(t)\right)$ pointwise converges to $z(t)$ for each $t \in[0, T]$, and $F(t, \cdot)$ is scalarly upper semicontinuous on $H$, one gets from the closure lemma [33, Theorem VI-4] that

$$
y(t) \in F(t, z(t)) \text { a.e. }
$$

Recall that, for any $n \geq 1, v_{h_{n}}$ is the unique solution to the following inclusion

$$
-\dot{v}_{h_{n}}(t) \in \partial \varphi\left(t, v_{h_{n}}(t)\right)+g_{n}(t) \text {, a.e. } t \in[0, T] \text {. }
$$

Since $\left(-\dot{v}_{h_{n}}-g_{n}\right)$ weakly converges to $(-\dot{z}-g)$ in $L_{H}^{2}([0, T])$, with $g(t)=f(t, h(t))+y(t)$, $y(t) \in F(t, z(t))$ a.e. by Proposition 3.1, one deduces that

$$
-\dot{z}(t) \in \partial \varphi(t, z(t))+g(t) \text { a.e. } t \in[0, T]
$$

that is,

$$
-\dot{z}(t) \in \partial \varphi(t, z(t))+f(t, h(t))+y(t), y(t) \in F(t, z(t)) \text { a.e. } t \in[0, T]
$$


It results that $-\dot{v}_{h}(t) \in \partial \varphi\left(t, v_{h}(t)\right)+f(t, h(t))+y(t)$ a.e. with $y(t) \in F\left(t, v_{h}(t)\right)$ a.e. and $v_{h}(0)=$ $u_{0} \in \operatorname{dom} \varphi(0, \cdot)$ so that $z=v_{h}$. Let us verify the continuity of $\Psi: \mathscr{X} \rightarrow \mathscr{X}$. Let $h_{n} \rightarrow h$. One has

$$
\begin{aligned}
\Psi\left(h_{n}\right)(t)-\Psi(h)(t) & =\int_{0}^{t} \frac{(t-s)^{\alpha-1}}{\Gamma(\alpha)} v_{h_{n}}(s) d s-\int_{0}^{t} \frac{(t-s)^{\alpha-1}}{\Gamma(\alpha)} v_{h}(s) d s \\
& =\int_{0}^{t} \frac{(t-s)^{\alpha-1}}{\Gamma(\alpha)}\left[v_{h_{n}}(s)-v_{h}(s)\right] d s .
\end{aligned}
$$

Since $\left(v_{h_{n}}(\cdot)-v_{h}(\cdot)\right) \rightarrow 0$ pointwise as $n \rightarrow \infty$ and is uniformly bounded $\left\|v_{h_{n}}(\cdot)-v_{h}(\cdot)\right\|_{\infty} \leq 2 L$, we conclude that

$$
\sup _{t \in[0, T]}\left\|\Psi\left(h_{n}\right)(t)-\Psi(h)(t)\right\| \leq \int_{0}^{T} \frac{(T-s)^{\alpha-1}}{\Gamma(\alpha)}\left\|v_{h_{n}}(\cdot)-v_{h}(\cdot)\right\|_{\infty} d s \rightarrow 0
$$

so that $\Psi\left(h_{n}\right)-\Psi(h) \rightarrow 0$ in $\mathscr{C}_{H}([0, T])$. The map $\Psi: \mathscr{X} \rightarrow \mathscr{X}$ is continuous and $\Psi(\mathscr{X})$ is relatively compact in $\mathscr{C}_{H}([0, T])$. Then, it follows from [42, 43] that $\Psi$ has a fixed point, say, $h=\Psi(h) \in \mathscr{X}$, which means

$$
h(t)=\Psi(h)(t)=x_{0}+\int_{0}^{t} \frac{(t-s)^{\alpha-1}}{\Gamma(\alpha)} v_{h}(s) d s,
$$

with

$$
\left\{\begin{array}{r}
-\dot{v}_{h}(t) \in \partial \varphi\left(t, v_{h}(t)\right)+f(t, h(t))+F\left(t, v_{h}(t)\right) \text { a.e. } \\
v_{h}(0)=u_{0} \in \operatorname{dom} \varphi(0, \cdot) .
\end{array}\right.
$$

Coming back to Lemma 5.1 and applying the above notation, one just finds a map $h$ satisfying

$$
\left\{\begin{array}{r}
-\frac{d}{d t}\left[{ }^{c} D^{\alpha} h(t)\right] \in \partial \varphi\left(t,\left[{ }^{c} D^{\alpha} h(t)\right]\right)+f(t, h(t))+F\left(t,\left[{ }^{c} D^{\alpha} h(t)\right]\right) \text { a.e. } \\
{ }^{c} D^{\alpha} h \text { is absolutely continuous } \\
\frac{d}{d t}\left[{ }^{c} D^{\alpha} h(\cdot)\right] \in L_{H}^{2}([0, T]) \\
h(0)=x_{0} .
\end{array}\right.
$$

Now, we address the following special case of Theorem 5.1 concerning the sweeping process involving fractional differential equation.

Theorem 5.2. Let $C:[0, T] \rightrightarrows H$ be a set-valued map satisfying $\left(H_{1}^{\prime}\right)-\left(H_{2}^{\prime}\right)$ of Remark 3.1. Let $f$ and $F$ satisfy the assumptions of Theorem 5.1. Then, for $\left(u_{0}, x_{0}\right) \in C(0) \times H$, there is an absolutely continuous map $u:[0, T] \rightarrow H$, and $a W_{H}^{\alpha, \infty}([0, T])$ map $x:[0, T] \rightarrow H$ such that

$$
\begin{gathered}
-\dot{u}(t) \in N_{C(t)}(u(t))+f(t, x(t))+F(t, u(t)) \text { a.e. } t \in[0, T], \\
{ }^{c} D^{\alpha} x(t)=u(t), t \in[0, T] \\
u(0)=u_{0} \in C(0), \\
x(0)=x_{0} \in H .
\end{gathered}
$$

Now, we give an application to optimal control. 
Theorem 5.3. Let $H=\mathbb{R}$. Let $\varphi:[0, T] \times H \rightarrow[0,+\infty]$ be a map satisfying $\left(H_{1}\right)-\left(H_{2}\right)$. Let $f:[0, T] \times H \rightarrow H$ be a Carathéodory map such that $\|f(t, x)\| \leq M$ for all $(t, x) \in[0, T] \times H$ and for some positive constant $M$. Let the cost functional $J:[0, T] \times H \times H \rightarrow[0,+\infty[$ be a measurable map such that $J(t, \cdot, \cdot)$ is lower semi-continuous on $H \times H$ for each $t \in[0, T]$, and $J(t, x, \cdot)$ is convex for each $(t, x) \in[0, T] \times H$. Then, the problem

$$
\min _{x \in \mathscr{X}} \int_{0}^{T} J\left(t, v_{x}(t), x(t)\right) d t
$$

has an optimal solution, where $v_{x}$ denotes the absolutely continuous solution associated with the control $x \in \mathscr{X}$ of the following evolution inclusion

$$
\begin{gathered}
-\dot{v}_{x}(t) \in \partial \varphi\left(t, v_{x}(t)\right)+f(t, x(t)) \text { a.e. } t \in[0, T], \\
{ }^{c} D^{\alpha} x(t)=v_{x}(t), t \in[0, T], \\
v_{x}(0)=u_{0} \in \operatorname{dom} \varphi(0, \cdot), \\
x(0)=x_{0} \in H .
\end{gathered}
$$

Proof. Let $\left(x_{n}(\cdot)\right)$ be a minimizing sequence of problem (5.13), that is,

$$
\lim _{n \rightarrow \infty} \int_{0}^{T} J\left(t, v_{x_{n}}(t), x_{n}(t)\right) d t=\inf _{x \in \mathscr{X}} \int_{0}^{T} J\left(t, v_{x}(t), x(t)\right) d t .
$$

By Theorem 5.1, one has $\sup _{n \in \mathbb{N}}\left\|\dot{v}_{x_{n}}\right\|_{L_{H}^{2}([0, T])}<\infty$ and the sequence $\left(v_{x_{n}}\right)$ is equi-absolutely continuous. Then, using similar arguments as in the proof of Theorem 5.1, we have that there exists a map $u \in W^{1,2}([0, T], H)$ such that $\left(v_{x_{n}}\right)$ uniformly converges to $u$ and $\left(\dot{v}_{x_{n}}\right)$ weakly converges to $\dot{u}$ in $L_{H}^{2}([0, T])$. Since $\mathscr{X}$ defined by $(5.10)$, that is,

$$
\mathscr{X}:=\left\{x_{g}:[0, T] \rightarrow H: x_{g}(t)=x_{0}+\int_{0}^{t} \frac{(t-s)^{\alpha-1}}{\Gamma(\alpha)} g(s) d s, g \in S_{L \bar{B}_{H}}^{1}, t \in[0, T]\right\},
$$

is a compact convex subset of the Banach space $\mathscr{C}_{H}([0, T])$ (see the proof of Theorem 5.1), then we may assume that $\left(x_{n}\right)$ uniformly converges to $x \in \mathscr{X}$. In particular, $\left(x_{n}\right)$ weakly converges in $L_{H}^{1}([0, T])$ to $x \in \mathscr{X}$. Moreover, as $f$ is bounded and continuous with respect to its second variable, along with the pointwise convergence of $\left(x_{n}(t)\right)$ to $x(t)$ for any $t \in[0, T]$, it follows from the dominated convergence theorem that

$$
\lim _{n \rightarrow \infty} \int_{0}^{T}\left\|f\left(t, x_{n}(t)\right)-f(t, x(t))\right\|^{2} d t=0 .
$$

By the lower semi-continuity theorem [44, Theorem 8.1.6] applied to the lower semi-continuous convex integral functional associated with $J$, one deduces

$$
\liminf _{n \rightarrow \infty} \int_{0}^{T} J\left(t, v_{x_{n}}(t), x_{n}(t)\right) d t \geq \int_{0}^{T} J(t, u(t), x(t)) d t .
$$

This along with (5.14) yields that

$$
\inf _{x \in \mathscr{X}} \int_{0}^{T} J\left(t, v_{x}(t), x(t)\right) d t=\int_{0}^{T} J(t, u(t), x(t)) d t .
$$

Recall that, for any $n \geq 1, v_{x_{n}}$ is the unique solution to the following inclusion

$$
\begin{gathered}
-\dot{v}_{x_{n}}(t) \in \partial \varphi\left(t, v_{x_{n}}(t)\right)+f\left(t, x_{n}(t)\right), \text { a.e. } t \in[0, T], \\
{ }^{c} D^{\alpha} x_{n}(t)=v_{x_{n}}(t), t \in[0, T],
\end{gathered}
$$




$$
\begin{gathered}
v_{x_{n}}(0)=u_{0} \in \operatorname{dom} \varphi(0, \cdot), \\
x_{n}(0)=x_{0} \in H .
\end{gathered}
$$

Using Proposition 3.1 yields

$$
-\dot{u}(t) \in \partial \varphi(t, u(t))+f(t, x(t)) \text { a.e. } t \in[0, T] .
$$

It results that $-\dot{v}_{x}(t) \in \partial \varphi\left(t, v_{x}(t)\right)+f(t, x(t))$ a.e. and $v_{x}(0)=u_{0} \in \operatorname{dom} \varphi(0, \cdot)$ so that $u=v_{x}$, where $v_{x}$ is the unique absolutely continuous solution associated to the control $x \in \mathscr{X}$ of the problem

$$
\begin{gathered}
-\dot{v}_{x}(t) \in \partial \varphi\left(t, v_{x}(t)\right)+f(t, x(t)) \text { a.e. } t \in[0, T], \\
{ }^{c} D^{\alpha} x(t)=v_{x}(t), t \in[0, T], \\
v_{x}(0)=u_{0} \in \operatorname{dom} \varphi(0, \cdot), \\
x(0)=x_{0} \in H .
\end{gathered}
$$

The proof of the proposition is then complete.

The following remark ends this section.

Remark 5.1. The existence result in Theorem 5.1 may find applications in optimal control theory by using Young measures, Filippov's theorems and the problems with delay in the line of recent works related to fractional differential and integral equations with applications; see, e.g., $[39,40,45]$. This needs further investigations and may be the subject of forthcoming research.

\section{Conclusion}

We provided a new existence result to the evolution problems of the form

$$
\left\{\begin{array}{l}
-\dot{x}(t) \in \partial \varphi(t, x(t))+F(t, x(t))+f(t, x(t)) \quad \text { a.e. } t \in I, \\
x(0)=x_{0} \in \operatorname{dom} \varphi(0, \cdot) .
\end{array}\right.
$$

An application to fractional differential equations with the Caputo fractional derivatives was given. There are many interesting points needed to further investigate, such as, functional differential inclusions, second-order evolution inclusions, Bolza, viscosity and relaxation problems in the theory of optimal control, variational inequalities and asymptotic theory with new perspectives.

\section{Acknowledgments}

The author is grateful to the referees for the useful suggestions which improved the final form of this paper.

\section{REFERENCES}

[1] J.J. Moreau, Rafle par un convexe variable, I, Sém. Anal. Convexe, Montpellier, Vol. 1 (1971), Exposé No. 15.

[2] M.D.P. Monteiro Marques, Differential Inclusions in Nonsmooth Mechanical Problems - Shocks and Dry Friction, Birkhauser Verlag, Basel, 1993.

[3] B. Cornet, Existence of slow solutions for a class of differential inclusions, J. Math. Anal. Appl. 96 (1983), 130-147.

[4] C. Henry, An existence theorem for a class of differential equations with multivalued right-hand side, J. Math. Anal. Appl. 41 (1973), 179-186.

[5] K. Addy, S. Adly, B. Brogliato, D. Goeleven, A method using the approach of Moreau and Panagiotopoulos for the mathematical formulation of non-regular circuits in electronics, Nonlinear Anal. Hybrid Syst. 1 (2007), 30-43. 
[6] B. Maury, J. Venel, A mathematical framework for a crowd motion model, C. R. Math. Acad. Sci. Paris 346 (2008), 1245-1250.

[7] H. Attouch, A. Damlamian, On multivalued evolution equations in Hilbert spaces, Israel J. Math., 12 (1972), 373-390.

[8] H. Benabdellah, C. Castaing, A. Salvadori, Compactness and discretization methods for differential inclusions and evolution problems, Atti. Sem. Math. Fis. Univ. Modena, XLV (1997), 9-51.

[9] H. Brézis, Opérateurs maximaux monotones et semi-groupes de contractions dans les espaces de Hilbert, Lecture Notes in Math., North-Holland, 1973.

[10] C. Castaing, A. Faik, A. Salvadori, Evolution equations governed by m-accretive and subdifferential operators with delay, Int. J. Appl. Math. 2 (2000), 1005-1026.

[11] C. Castaing, M.D.P. Monteiro Marques, Evolution problems associated with nonconvex closed moving sets with bounded variation, Port. Math. 53 (1996), 73-78.

[12] C. Castaing, M.D.P. Monteiro Marques, S. Saïdi, Evolution problems with time-dependent subdifferential operators, Adv. Math. Econ. 23 (2019), 1-39.

[13] C. Castaing, A. Salvadori, L. Thibault, Functional evolution equations governed by nonconvex sweeping process, J. Nonlinear Convex Anal. 2 (2001), 217-241.

[14] J.F. Edmond, L. Thibault, BV solution of nonconvex sweeping process differential inclusion with perturbation, J. Differential Equations 226 (2006), 135-159.

[15] S. Hu, N.S. Papageorgiou, Handbook of multivalued analysis. Vol. II, volume 500 of Mathematics and its Applications, Kluwer Academic Publishers, Dordrecht, 2000.

[16] N. Kenmochi, Solvability of nonlinear evolution equations with time-dependent constraints and applications, Bull. Fac. Educ. Chiba Univ. 30 (1981) 1-87.

[17] M. Kubo, Characterisation of a class of evolution operators generated by time dependent subdifferential, Funkcial. Ekvac. 32 (1989), 301-321.

[18] J.C. Peralba, Équations d'évolution dans un espace de Hilbert, associées à des opérateurs sous-différentiels, Thèse de doctorat de spécialité, Montpellier, (1973).

[19] S. Saïdi, L. Thibault, M. Yarou, Relaxation of optimal control problems involving time dependent subdifferential operators, Numer. Funct. Anal. Optim. 34 (2013), 1156-1186.

[20] S. Saïdi, M.F. Yarou, Set-valued perturbation for time dependent subdifferential operator, Topol. Methods Nonlinear Anal. 46 (2015), 447-470.

[21] A.A. Tolstonogov, Properties of attainable sets of evolution inclusions and control systems of subdifferential type, Sib. Math. J. 45 (2004), 763-784.

[22] Y. Yamada, On evolution equations generated by subdifferential operators, J. Math. Sci. Univ. Tokyo 23 (1976), 491-515.

[23] D. Azzam-Laouir, W. Belhoula, C. Castaing, M.D.P. Monteiro Marques, Multi-valued perturbation to evolution problems involving time dependent maximal monotone operators, Evol. Equ. Control Theory 1 (2020), 219-254.

[24] M. Kunze and M.D.P. Monteiro Marques, BV solutions to evolution problems with time-dependent domains, Set-Valued Anal. 5 (1997), 57-72.

[25] E. Vilches, B.T. Nguyen, Evolution inclusions governed by time-dependent maximal monotone operators with a full domain, Set-Valued Var. Anal. 28 (2020), 569-581.

[26] A.A. Vladimirov, Nonstationary dissipative evolution equations in Hilbert space, Nonlinear Anal. 17 (1991), 499-518.

[27] C. Castaing, T. X. Dùc Ha and M. Valadier, Evolution equations governed by the sweeping process, Set-Valued Anal. 1 (1993), 109-139.

[28] J.J. Moreau, Evolution problem associated with a moving convex set in a Hilbert space, J. Differential Equations, 26 (1977), 347-374.

[29] M. Valadier, Quelques résultats de base concernant le processus de la rafle, Sém. Anal. Convexe, Montpellier, 3 (1988), 1-30.

[30] D. Azzam-Laouir, A. Makhlouf, L. Thibault, On perturbed sweeping process, Appl. Anal. 95 (2016), 303-322.

[31] F. Nacry, Perturbed BV sweeping process involving prox-regular sets, J. Nonlinear Convex Anal. 18 (2017), 1619-1651. 
[32] F. Nacry, Truncated nonconvex state-dependent sweeping process: implicit and semi-implicit adapted Moreau's catching-up algorithms, J. Fixed Point Theory Appl. 20 (2018).

[33] C. Castaing, M. Valadier, Convex analysis and Measurable Multifunctions, Lecture Notes in Math., 580, Springer-Verlag Berlin Heidelberg, 1977.

[34] J.J. Moreau, Rétraction d'une multiapplication, Sém. Anal. convexe, Montpellier (1972), Exposé 13.

[35] A.A. Tolstonogov, Sweeping process with unbounded nonconvex perturbation, Nonlinear Anal. 108 (2014), 291-301.

[36] E. Klein, A. C. Thompson, Theory of Correspondences, Wiley, New York, 1984.

[37] A.A. Kilbas, H.M. Srivastava, J.J. Trujillo, Theory and Applications of Fractional Differential Equations, Math. Stud. 204, North Holland, 2006.

[38] I. Podlubny, Fractional Differential Equation, Academic Press, San Diego (1999).

[39] M. Bergounioux and L. Bourdin, Pontryagin maximum principle for general Caputo fractional optimal control problems with Bolza cost and terminal constraints, ESAIM Control Optim. Calc. Var. 26 (2020).

[40] C. Castaing, C. Godet-Thobie, F.Z. Mostefai, On a fractional differential inclusion with boundary conditions and application to subdifferential operators, J. Nonlinear Convex Anal. 18 (2017), 1717-1752.

[41] A.A. Kilbas, B Bonilla, J.J. Trukhillo, Existence and uniqueness theorems for nonlinear fractional differential equations, Demonstr. Math. XXXIII (2000), 583-602.

[42] A. Idzik, Almost fixed points theorems, Proc. Amer. Math. Soc. 104 (1988), 779-784.

[43] S. Park, Fixed points of approximable or Kakutani maps, J. Nonlinear Convex Anal. 7 (2006), 1-17.

[44] C. Castaing, P. Raynaud de Fitte and M. Valadier, Young measures on topological spaces with applications in control theory and probability theory, Kluwer Academic Publishers, Dordrecht, 2004.

[45] C. Castaing, C. Godet-Thobie, P.D. Phung, L.X. Truong, On fractional differential inclusions with nonlocal boundary conditions, Fract. Calc. Appl. Anal. 22 (2019), 444-478. 